\title{
Crowding changes appearance systematically in peripheral, amblyopic, and developing vision
}

Kalpadakis-Smith, A.V. ${ }^{1}$, Tailor, V.K. ${ }^{1,2,3}$, Dahlmann-Noor, A.H. ${ }^{2,3}$, \& Greenwood, J.A. ${ }^{1}$

${ }^{1}$ Experimental Psychology, University College London, London, UK

${ }^{2}$ NIHR Biomedical Research Centre @ Moorfields Eye Hospital, London, UK

${ }^{3}$ Moorfields Eye Hospital NHS Foundation Trust, London, UK

Keywords: $\quad$ Crowding, amblyopia, development, peripheral vision, orientation.

Corresponding author: John A. Greenwood

Email:

Website: http://eccentricvision.com 


\section{Abstract}

Visual crowding is the disruptive effect of clutter on object recognition. Although most prominent in adult peripheral vision, crowding also disrupts foveal vision in typically-developing children and those with strabismic amblyopia. Do these crowding effects share the same mechanism? Here we exploit observations that crowded errors in peripheral vision are not random: target objects appear either averaged with the flankers (assimilation), or replaced by them (substitution). If amblyopic and developmental crowding share the same mechanism then their errors should be similarly systematic. We tested foveal vision in children aged 3-9 years with typical vision or strabismic amblyopia, and peripheral vision in adults. The perceptual effects of crowding were measured by requiring observers to adjust a reference stimulus to match the perceived orientation of a target 'Vac-Man' element. When the target was surrounded by flankers that differed by $\pm 30^{\circ}$, adults and children reported orientations between the target and flankers (assimilation). Errors were reduced with $\pm 90^{\circ}$ differences, but primarily matched the flanker orientation (substitution) when they did occur. A population pooling model of crowding successfully simulated this pattern of errors in all three groups. We conclude that the perceptual effects of amblyopic and developing crowding are systematic and resemble the near periphery in adults, suggesting a common underlying mechanism. 


\section{Introduction}

Clutter can significantly disrupt the recognition of objects that are otherwise readily identified in isolation - a phenomenon known as visual crowding (Levi, 2008; Whitney \& Levi, 2011). In the typical adult visual system, crowding is most pronounced in peripheral vision, where the recognition of a target can be hindered by flanking objects separated by as much as half the target eccentricity (Bouma, 1970), and minimal in the fovea (Toet \& Levi, 1992; Danilova \& Bondarko, 2007; Coates, Levi, Touch, \& Sabesan, 2018). Crowding also affects vision in amblyopia, a developmental disorder of vision characterised by reduced acuity in one eye despite optical correction (McKee, Levi, \& Movshon, 2003). When amblyopia is associated with strabismus (ocular misalignment), foveal vision in the affected eye is significantly impaired by the presence of nearby flankers (Flom, Weymouth, \& Kahneman, 1963; Levi \& Klein, 1985; Greenwood et al., 2012). Foveal elevations in crowding have also been found in children with typical vision up to the age of 11 years (Jeon, Hamid, Mauer, \& Lewis, 2010; Greenwood et al., 2012). These elevations would have a range of functional consequences, given for instance the correlation between crowding and reading ability (Martelli, Di Filippo, Spinelli, \& Zoccolotti, 2009). However, although it is clear that crowding occurs in these three instances - the typical adult periphery, the amblyopic fovea, and the developing fovea - it is unclear whether the underlying mechanism is the same.

In the typical adult periphery, the placement of flankers near to a target hinders its identification. The spatial extent of crowding can be defined as the separation required to remove this disruptive effect (Bouma, 1970; Toet \& Levi, 1992), a property that is invariant with target size (Levi, Hariharan, \& Klein, 2002b; Tripathy \& Cavanagh, 2002). Peripheral crowding is also selective for the similarity between the target and flankers in visual dimensions such as orientation and contrast polarity, with target identification more strongly disrupted when flankers are similar to the target than when they are dissimilar (Kooi, Toet, Tripathy, \& Levi, 1994; Wilkinson, Wilson, \& Ellemberg, 1997; Chung, Levi, \& Legge, 2001). In the amblyopic fovea, crowding is similarly reduced by an increase in target-flanker separation (Levi, Hariharan, \& Klein, 2002a; Hariharan, Levi, \& Klein, 2005), with an extent that is also largely invariant to target size (Levi, Hariharan, \& Klein, 2002a). Amblyopic crowding may however be less dependent on target-flanker similarity flankers dissimilar to the target in polarity, contrast, and orientation have been found to be equally disruptive as flankers similar to the target along these dimensions (Levi, Hariharan, \& Klein, 2002a; Hariharan, Levi, \& Klein, 2005). These variations in the characteristics of crowding cast doubt on the possibility of a common mechanism for peripheral vision and the amblyopic fovea. Although crowding in the developing fovea is also dependent on target-flanker separation (Greenwood et al., 2012), its dependence on target size or target-flanker similarity is unclear. 
Much of our understanding of the mechanisms underlying crowding in the typical adult periphery derives from measurements of the errors that observers make when reporting the identity of a crowded target. Observers have been found to report either the identity of one of the flankers surrounding the target (Strasburger, Harvey, \& Rentschler, 1991; Strasburger, 2005), or an intermediate identity close to the target-flanker average (Parkes et al., 2001; Greenwood, Bex, \& Dakin, 2009). The finding that target patches of noise can similarly adopt the perceived orientation of the flankers (Greenwood, Bex, \& Dakin, 2010) suggests that these errors are not simply the result of decisional bias. Rather, they represent a change in the perceived identity of the target to more closely resemble the flankers, indicating that peripheral crowding has systematic perceptual effects.

A range of models has been put forward to account for the systematic shift in the identity of crowded targets. Substitution models (Ester, Klee, \& Awh, 2014; Ester, Zilber, \& Serences, 2015) argue that errors emerge due to the substitution of a flanker into the target location, leading observers to report the flanker identity. This substitution is either attributed to the increased positional uncertainty of peripheral vision (Wolford, 1975; Krumhansl \& Thomas, 1977), or unfocused spatial attention (Strasburger, Harvey, \& Rentschler, 1991; Strasburger, 2005). On the other hand, 'pooling' or averaging models (Parkes et al., 2001; Greenwood, Bex, \& Dakin, 2009; Dakin, Cass, Greenwood, \& Bex, 2010) posit that crowding is the compulsory integration of target and flanker signals, resulting in observers perceiving an average or intermediate feature (e.g. orientation) of the target and flankers. However, each of these model types focuses on distinct types of errors: either flanker reports ('substitution errors') or reports of intermediate identities between the target and flankers ('assimilation errors').

Population pooling models (van den Berg, Roerdink, \& Cornelissen, 2010; Harrison \& Bex, 2015; Greenwood \& Parsons, 2020) propose a more general framework for the perceptual effects of peripheral crowding. Harrison and Bex (2015) used an orientation-matching task where observers matched the orientation of a reference Landolt- $C$ to that of a crowded target in the periphery. When the target was surrounded by flankers that differed by $45^{\circ}$ or less, observers reported orientations between the target and the flanker values (assimilation errors). When the difference from the target was $90^{\circ}$ and above, errors more closely matched the flanker orientation (substitution errors). Rather than invoking separate substitution or averaging mechanisms, Harrison and Bex (2015) account for both error types using a population pooling model that takes a weighted combination of population responses to the target and flankers. Similar approaches have been applied more generally to explain crowding with letters (Freeman, Chakravarthi, \& Pelli, 2012) and faces (Kalpadakis-Smith, Goffaux, \& Greenwood, 2018). Higher-dimensional pooling approaches have also been developed, which depict crowding as an over-application of summary statistics 
across visual space (Balas, Nakano, \& Rosenholtz, 2009; Freeman \& Simoncelli, 2011; Keshvari \& Rosenholtz, 2016). Their generality allows the consideration of these crowding effects in a range of naturalistic tasks (Rosenholtz, Yu, \& Keshvari, 2019), though quantitative predictions of these highdimensional models are more difficult to discern for specific paradigms.

Although population pooling models can account for the systematic perceptual effects of peripheral crowding, their applicability to amblyopia is unknown. Given the plethora of deficits in visual function observed in the affected eye (McKee, Levi, \& Movshon, 2003), the basis of amblyopic crowding could in fact differ substantially. In addition to the definitive acuity deficit, vision in the affected eye of observers with amblyopia is characterised by increased positional uncertainty (Levi \& Klein, 1985). This uncertainty could produce confusions of the flanker for the target, making a predominance of substitution errors. Alternatively, crowded errors may arise due to perceptual distortions that affect the amblyopic eye. Observers with strabismic amblyopia show considerable distortions when reconstructing circle stimuli, including shrinkage, expansion, and torsion of specific regions (Pugh, 1958; Sireteanu, Lagreze, \& Constantinescu, 1993). Although these distortions are consistent over time, they vary across observers and visual field location (Barrett et al., 2003). If these distortions underlie the perceptual effects of amblyopic crowding, then errors would not be systematic but random (depending on the particular conjunction of the distortion type and stimulus), suggesting a distinct mechanism from peripheral crowding.

Even less is known about the mechanism of foveal crowding during development. Although the extent of foveal crowding has been found to be greater in typically developing children than in adults (Atkinson \& Braddick, 1983; Atkinson, Anker, Evans, \& McIntyre, 1987; Jeon et al., 2010; Greenwood et al., 2012), the perceptual effects of developing crowding have not been investigated. Studies have however demonstrated that children make a disproportionate amount of random errors in psychophysical tasks relative to adults (Witton, Talcott, \& Henning, 2017; Manning, Jones, Dekker, \& Pellicano, 2018). These errors are frequently made in low difficulty "catch" trials (Treutwein, 1995) and have been attributed to attentional lapses and underdeveloped short-term memory (Witton, Talcott, \& Henning, 2017; Manning et al., 2018). Both of these factors would produce random errors that could dominate responses to the identity of a target object in crowding paradigms. The same could be true of children with amblyopia. In both cases, the observed elevations in foveal crowding could therefore reflect quite distinct processes to the systematic perceptual effects observed in peripheral vision.

To investigate the perceptual effects of amblyopic and developing crowding, we tested children aged 3-9 years with strabismic amblyopia or typical vision, and adults with typical vision. We used an orientation-matching task similar to the one used by Harrison and Bex (2015), here with Landolt-C elements presented foveally to children and in peripheral vision to adults. Given the 
timing constraints in testing children (due to shorter attention spans), we tested $\pm 30^{\circ}$ and $\pm 90^{\circ}$ target-flanker orientation differences. The $\pm 30^{\circ}$ flanker differences were selected to test whether children show the same systematic shift of responses to intermediate orientations between the target and flankers (assimilation errors) observed in the periphery. Because orientation differences below $90^{\circ}$ are less able to distinguish between assimilation and substitution errors (Harrison \& Bex, 2015), we chose $\pm 90^{\circ}$ flanker differences to further constrain the underlying mechanisms. These $90^{\circ}$ orientation differences have also been shown to reduce the effect of crowding in the periphery relative to smaller orientation differences (Hariharan, Levi, \& Klein, 2005; Harrison \& Bex, 2015), allowing us to examine the selectivity for target-flanker similarity in amblyopic and developing crowding. We further probed the possibility of a common mechanism by simulating the observed perceptual effects with a population pooling model of crowding, with comparison to simulations of a model that simply added noise to the orientation judgements.

If there is a common mechanism that underlies amblyopic, developing, and peripheral crowding, each of these instances should show the same systematic effects on target appearance as observed in the adult periphery. That is, children with amblyopia and typical vision should make either assimilation and substitution errors, depending on the target-flanker orientation difference. In contrast, distinct mechanisms for crowding in strabismic amblyopia and developing vision may produce random errors, either because crowding affects the appearance of the target in a nonsystematic manner or due to attentional lapses.

\section{Methods}

\section{Design}

Both children and adults completed three tasks. Acuity was measured first to determine the minimum target size at which each observer could judge the gap orientation of the Landolt-C element. Crowded acuity was then measured to determine the extent of the spatial zone of crowding around the target location. These tasks, adapted from Greenwood et al. (2012), were used to set the parameters for the third orientation-matching task, and in particular to ensure both that the target size was above acuity limits and that flanker locations were within the spatial region of crowding. The third orientation-matching task was adapted from that used by Harrison \& Bex (2015), which allowed us to measure the perceptual effects of crowding.

\section{Observers}

\section{Children}

40 children between 3-9 years of age were tested, divided into two groups: those with typical vision ( $n=20$, mean $=73.2$ months), and those with strabismic amblyopia $(n=20$, mean $=$ 
72.1 months). Sample sizes were derived from prior work (Greenwood et al., 2012), with all children tested at the Richard Desmond Children's Eye Centre at Moorfields Eye Hospital (London, UK).

Prior to the study, children underwent a full orthoptic assessment to ensure they met our inclusion and exclusion criteria. In the typically developing group, children were selected to have a best-corrected visual acuity of 0.1 logMAR (logarithm of the minimum angle of resolution) or better in both eyes, as measured by Thomson V2000 acuity charts, in the absence of any pre-existing visual or neurological deficits. Clinical details for the children with typical vision can be found in Appendix A.

For children with strabismic amblyopia, inclusion was based on the presence of amblyopia, as indicated by a two-line difference in best-corrected logMAR acuity between the eyes, as well as heterotropia (deviation of the optical axes) that could be either esotropia (inward deviation) or exotropia (outward). Children with additional visual deficits (e.g. macular dystrophies) and developmental or neurological deficits (e.g. autism) were excluded. We did not exclude cases of joint anisometropia and strabismus. Clinical details for these children are shown in Appendix A.

Three children with amblyopia did not complete all experimental tasks and were excluded from the analysis. They are not included in the tallies above. The experimental procedures were performed with the informed consent of children and their parents and were approved by the Health Research Authority of the UK National Health Service.

Adults

10 adults were tested ( 4 males, $M=28.7$ years, range $24-35$ years). All had a best-corrected visual acuity of 0 logMAR or better. As indicated by their own report, none had amblyopia or strabismus, nor any history of binocular dysfunction.

\section{Apparatus}

\section{Children}

Experiments were programmed using Matlab (The Mathworks, Ltd., Cambridge, UK) and run on a Dell PC using PsychToolbox (Brainard, 1997; Pelli, 1997). Stimuli were presented on an ASUS VG278HE LCD monitor, with $1920 \times 1080$ resolution and $120 \mathrm{~Hz}$ refresh rate. The monitor was calibrated using a Minolta photometer and linearised in software, to give a maximum luminance of $150 \mathrm{~cd} / \mathrm{m}^{2}$. A second Dell UltraSharp 2208WFP monitor, with $1680 \times 1050$ resolution and $75 \mathrm{~Hz}$ refresh rate, was positioned above the first. In the acuity and crowding-extent tasks, this second monitor was used to display a running tally of points children received by playing the games. In the orientation-matching task it displayed the response stimulus.

Figure $1 \mathrm{~A}$ shows the experimental setup for the children. Children wore stereo-shutter glasses (nVidia Corp., Santa Clara, CA) alternating at $120 \mathrm{~Hz}$. These glasses were used to present the stimuli monocularly. The glasses were custom-fit into a ski-mask frame to allow children to 
wear them comfortably over their optical correction. Children were seated $3 \mathrm{~m}$ from the screen. For the acuity and crowding-extent tasks, the experimenter recorded the children's responses using the keyboard. For the orientation-matching task, a Griffin Powermate response dial was used by the children to rotate the response element and register their responses.

\section{Adults}

Adults completed the same three tasks as children, run on a Viglen PC and presented on a Sony GDM-FW900 cathode ray tube (CRT) monitor with $2304 \times 1440$ resolution and $80 \mathrm{~Hz}$ refresh rate. The monitor was calibrated and linearised to give a maximum luminance of $122 \mathrm{~cd} / \mathrm{m}^{2}$. For the acuity and crowding-extent tasks, observers registered their response using a keyboard. Responses in the orientation-matching task were made with a Griffin Powermate dial.

Observers were seated $50 \mathrm{~cm}$ from the monitor, with a head-and-chin rest used to minimise movement. Stimuli were presented monocularly to the dominant eye, with an eye-patch covering the non-dominant eye. Eye-dominance was established using the Miles test (Miles, 1928). Figure 1B depicts this experimental setup.

\section{Stimuli and Procedures}

\section{Children}

The three tasks involved five video-game characters adapted from a previous study (Greenwood et al., 2012): Vac-Man (Visual Acuity Man) and four ghosts. Vac-Man was a circle with a horizontal gap for a "mouth" in its centre, resembling a filled-in Landolt-C. The size of the mouth was one-fifth of the stimulus diameter, similar to Sloan letters (Sloan, 1959). Vac-Man was the centrally located target stimulus in all three tasks, rendered in black at $90 \%$ Weber contrast against a mid-grey $\left(45 \mathrm{~cd} / \mathrm{m}^{2}\right)$ background. Vac-Man also served as flanker and response stimuli in the orientation-matching task. The ghost characters acted either as colour aids for the identification of Vac-Man's orientation in the acuity task (as in Figure 1A), or achromatic flanker stimuli in the crowding-extent task. The gap for each of the ghosts' "legs" was also one-fifth of the stimulus diameter.

All children began with the acuity task, where they were asked to report which of the ghosts Vac-Man was facing (4 Alternative Forced Choice, 4AFC). Each ghost had a distinct colour (green above, red to the right, orange below, and green to the left) and moved slowly along the monitor edges at a large separation from the target (as in Figure 1A) to minimise the chance of any crowding with the target. Children could report either the colour of the ghost, or its location verbally or by pointing. Pictures of the ghosts were placed at the monitor edges to aid children's reports. Normal colour-naming abilities were checked using the stimuli prior to participation. Feedback was given after each trial through brief animations, with Vac-Man smiling for correct 
bioRxiv preprint doi: https://doi.org/10.1101/2021.11.30.470647; this version posted December 2, 2021. The copyright holder for this preprint (which was not certified by peer review) is the author/funder, who has granted bioRxiv a license to display the preprint in perpetuity. It is made available under aCC-BY-NC-ND 4.0 International license.

responses and frowning when incorrect. A longer animation was presented after 3 correct responses in which Vac-Man ate a ghost (see Figure 1C). Children had unlimited response time.

A.
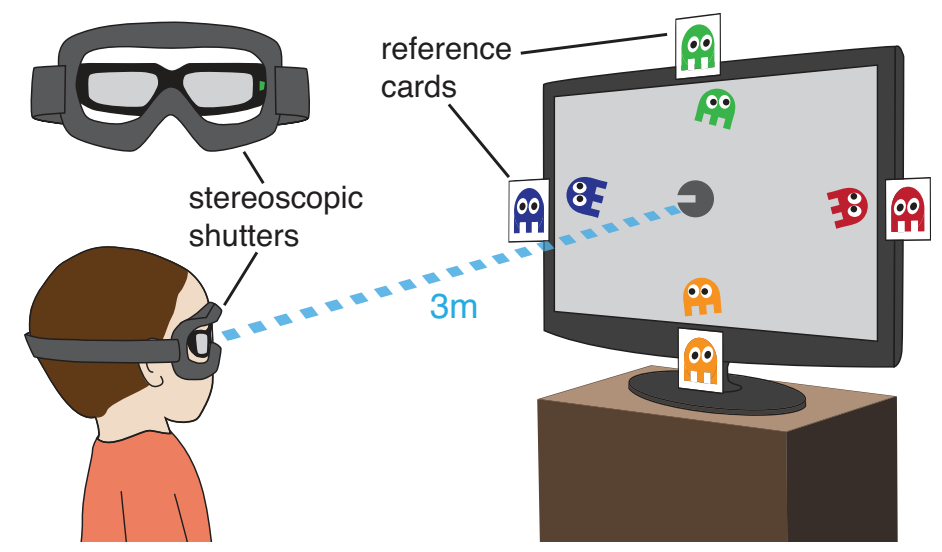

B.

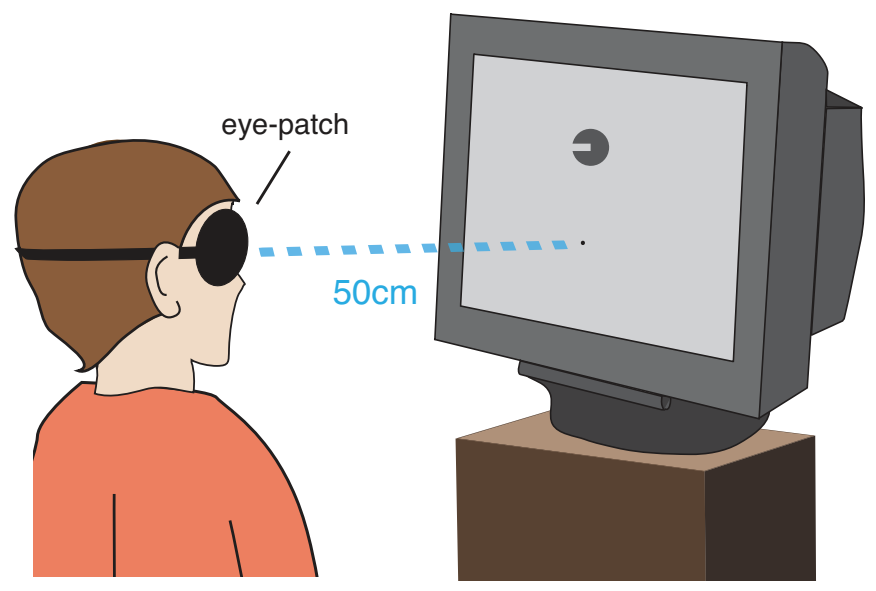

C.

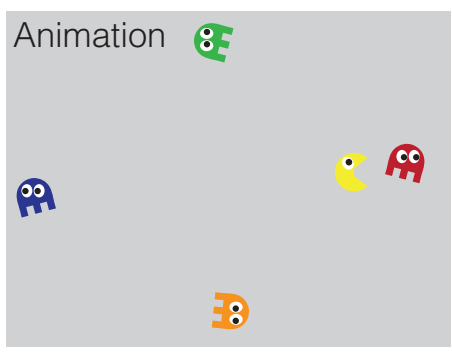

D.

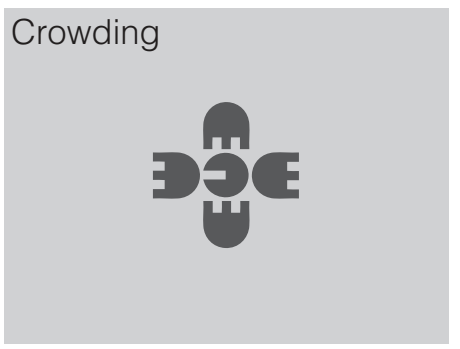

E.

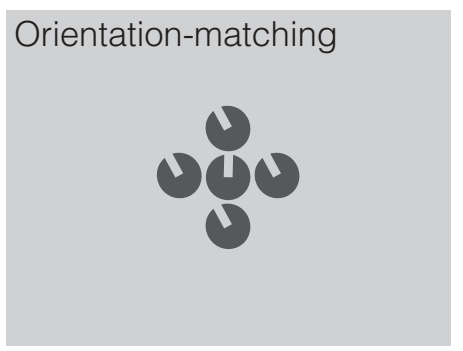

Figure 1. Apparatus and stimuli.

A. For children, stimuli were viewed through stereoscopic shutter glasses mounted in a ski mask and presented on a 3Dcompatible monitor at $3 \mathrm{~m}$ distance. An example trial of the acuity task is depicted, where children reported the colour of the ghost that Vac-Man was facing. Coloured cards of the ghosts on the monitor edges helped children select the ghost. B. Adults viewed the monitor from $50 \mathrm{~cm}$, wearing an eye patch over their non-dominant eye. C. An example frame from the "reward animation", presented every three correct trials. D. Illustration of the stimuli in the crowding-extent task. Ghost flankers were presented at random orientations at a fixed relative separation (1.1× stimulus diameter), with their absolute separation varied by QUEST. E. Illustration of stimuli in the orientation matching task. Here, flankers were filledin Landolt-Cs, similar to the target, presented with the same orientation difference at a fixed separation.

In the subsequent crowding-extent task, the four ghosts became flankers surrounding VacMan, each achromatic in order to match the target and increase the strength of crowding through target-flanker similarity (Kooi et al., 1994). Flanker ghosts were located above, below, left, and right of Vac-Man, with each ghost randomly oriented in 1 of 4 cardinal orientations. Children made the same 4AFC judgement as the acuity task, aided by full-colour reference cards of the ghosts on the monitor edges. 
Acuity thresholds were measured by varying the overall size of Vac-Man, and thus the visibility of the mouth gap to indicate his orientation. Size was varied using a QUEST staircase procedure (Watson \& Pelli, 1983) set to converge at $62.5 \%$ correct performance. These gap-size thresholds set the lower bound for the size used in the orientation-matching task. The spatial extent of crowding was also measured by varying Vac-Man size, with QUEST converging at a higher level of $80 \%$ correct performance. Flanker ghosts were scaled similarly, with the centre-tocentre separation between the target and flankers scaled at $1.1 \times$ target diameter, a value recommended as efficient for the measurement of crowding extent (Song, Levi, \& Pelli, 2014). Although this method confounds size and separation by varying both, the extent of amblyopic and peripheral crowding are limited by centre-to-centre separation and not target size (Hariharan, Levi, \& Klein, 2005). As such, it is only the variations in centre-to-centre separation that should affect the strength of crowding, and thus the measurement of its extent (Levi, Song, \& Pelli, 2007; Song, Levi, \& Pelli, 2014). The resulting gap-size threshold gave the upper bound for the sizes and targetflanker separations used in the orientation-matching task, ensuring that stimuli were placed within the spatial extent of crowding.

The QUEST routine used for both acuity and crowding-extent tasks was tailored to suit children in three ways. First, to begin the task children were given 3 practice trials with a target gap-size set at twice their acuity value measured during orthoptic testing. Second, easier trials were presented on every fifth trial by selecting a gap size at twice the current QUEST threshold estimate. This minimised the frustration arising from the presentation of numerous trials near threshold. Third, an exit criterion was added to reduce the time taken for threshold estimation: if the standard deviation of the threshold estimated by QUEST for the preceding 8 trials was below 0.03 log units, the experimenter was given the option to exit the task. Otherwise, the QUEST terminated after 60 trials (30 for each eye). The average number of trials needed to estimate threshold, excluding practice trials, was 44 for acuity and 46 for the crowding-extent task. Both eyes were tested in one experimental run, with separate QUEST staircases for each eye running simultaneously and selected at random on each trial. The output of each QUEST staircase gave the size of Vac-Man's mouth in degrees of visual angle at the predefined threshold of $62.5 \%$ correct.

The final orientation-matching task allowed us to investigate the perceptual effects of crowding. Four achromatic "imposter" Vac-Men surrounded the "real" target Vac-Man in each cardinal direction. Both target and flanker Vac-Men were at $90 \%$ Weber contrast. Here, a second response Vac-Man was presented on the response screen, twice the size of the target to ensure visibility. Children were asked to use the response dial to rotate the Vac-Man on the response screen to appear the same as the real Vac-Man on the main monitor. They had unlimited time to 
respond. On each trial, the orientation of the target varied randomly between $\pm 45^{\circ}$ from vertical. The four flankers were matched in orientation, all of them differing from the target by either $\pm 30^{\circ}$ or $\pm 90^{\circ}$. This resulted in five flanker conditions: unflanked (target in isolation), or surrounded by flankers with a difference of either $+30^{\circ}$ (counter-clockwise), $-30^{\circ}$ (clockwise), $+90^{\circ}$, or $-90^{\circ}$ from the target. 12 trials were tested for each condition, resulting in 60 trials in total for the orientationmatching task. When children's responses deviated from the orientation of the target by more than $\pm 35^{\circ}$, they received feedback in the form of a frowning Vac-Man, whereas when they responded within that range, Vac-Man smiled. This was done to maintain children's engagement in the task and reward them for participating.

Stimulus sizes in the matching task were determined individually for each child, both to ensure that Vac-Man was visible (i.e. above the acuity limit) and crowded (i.e. with flankers within the spatial extent of crowding). A multiple of the gap-size acuity threshold was thus used, constrained by the crowding-extent values. We aimed to present stimuli at $3 \times$ the acuity threshold, though where this gave target-flanker separations that exceeded the crowding extent for that child, lower values were used (descending from $2.5 \times, 2 \times$, or $1.5 \times$ the acuity threshold). For the amblyopic children, three were tested with sizes $3 \times$ acuity thresholds, seven with $2.5 \times$, five with $2 \times$, and five with $1.5 \times$. For those with typical vision, one child was tested with $2.5 \times$, six with $2 \times$, and thirteen with $1.5 \times$.

Adults

Adults completed the same three tasks as the children with the same stimuli (minus reward animations), albeit with the addition of a Gaussian fixation point near the bottom of the monitor. Stimuli were presented monocularly to the dominant eye at four eccentricities: $2.5^{\circ}, 5^{\circ}, 10^{\circ}$, and $15^{\circ}$ in the upper visual field.

On each trial of the acuity and crowding-extent tasks, the fixation point first appeared for $500 \mathrm{~ms}$. This was followed by the target, either in isolation (acuity task) or surrounded by the ghost flankers (crowding-extent task) for $500 \mathrm{~ms}$. A circular $1 / \mathrm{f}$ noise mask with a diameter of $1 / 3$ the target eccentricity was then presented for $250 \mathrm{~ms}$. A different mask was presented on each trial. After the presentation of the mask, observers had unlimited time to make a 4AFC response on the target orientation. A 500ms inter-trial interval followed, with the fixation dot on screen. Each staircase consisted of 45 trials, with observers completing two staircases per eccentricity. For each observer, acuity and crowding-extent values were taken from the average gap-size threshold from the two staircases at each eccentricity.

For the orientation-matching task, stimuli were identical to the children's version (Figure 1E). Stimuli were presented at a size $3 \times$ the acuity threshold, which gave target-flanker separations that fell within the crowding extent for all observers. The trial presentation sequence was similar to 
the acuity and crowding tasks, with a fixation dot appearing for $500 \mathrm{~ms}$, followed by the target for $500 \mathrm{~ms}$. The target was either presented in isolation or surrounded by flankers of $\mathrm{a} \pm 30^{\circ}$ or $\pm 90^{\circ}$ orientation difference. A $1 / \mathrm{f}$ noise mask was then presented for $250 \mathrm{~ms}$, at which point a reference stimulus identical to the target appeared at fixation at a random orientation. The size of the reference matched the target. Observers had unlimited time to adjust the reference stimulus to match the orientation of the previously presented target. Adults completed 5 blocks of 100 trials per eccentricity, resulting in a total of 2000 trials per observer. In each block, 20 trials were included for each of the 5 flanker conditions. Blocks for each eccentricity were interleaved to counter any practice effects. Observers received auditory feedback in the form of a beep when their estimate of the target orientation was offset by more than $\pm 35^{\circ}$. All other parameters were identical to the children's version of the tasks.

\section{Results}

\section{Acuity and Crowding Extent}

The acuity and crowding-extent tasks each gave a measure of the gap size of the Vac-Man target required for performance to reach a particular point ( $62.5 \%$ for acuity; $80 \%$ for crowding). For acuity, the gap size was the value of interest. For crowding, the spatial extent of crowding was calculated as the radius from the centre of the target to the centre of one flanker. This centre-tocentre separation was equal to the target diameter (which was five times the gap size) multiplied by 1.1 (the relative separation between elements). Here we consider the adult results first, followed by the children.

\section{Adult Periphery}

Gap-size (acuity) thresholds for the four eccentricities can be seen in Figure 2A. Thresholds increased with eccentricity, averaging 2.3 arcmins at $2.5^{\circ}$ eccentricity, 3.7 arcmins at $5^{\circ}, 7$ arcmins at $10^{\circ}$, and 11.4 arcmins at $15^{\circ}$. A one-way ANOVA revealed a significant effect of eccentricity, $(F[1.47,13.20]=110.34, P<.0001$, Greenhouse-Geisser corrected), demonstrating the well-known reduction of acuity in the periphery. 
A.

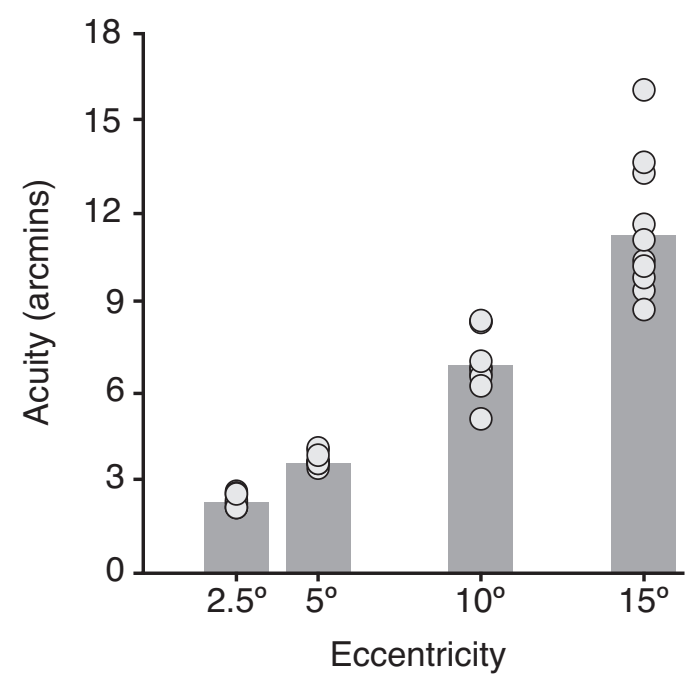

B.

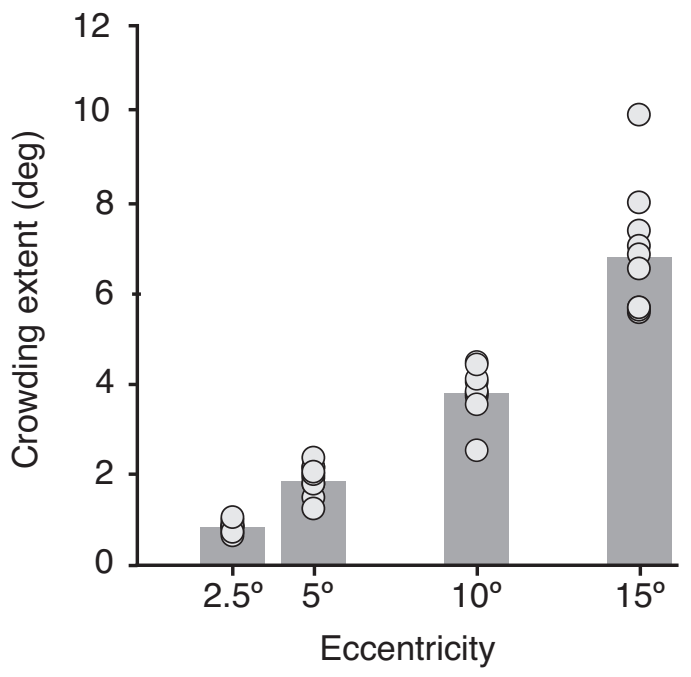

Figure 2. Acuity and crowding in the adult periphery.

\begin{abstract}
A. Acuity measured as gap-size thresholds (in minutes of arc) for adult observers in peripheral vision. Dots indicate values for each observer, with the mean shown as a bar $(n=10)$. B. The spatial extent of crowding, measured as the centre-to-centre separation (in degrees of visual angle) between the target and flankers, plotted as in panel A.
\end{abstract}

The spatial extent of crowding at each eccentricity is presented in Figure 2B. Crowding also increased with eccentricity, though with a vast difference in scale from acuity, averaging $0.82^{\circ}$ at $2.5^{\circ}, 1.88^{\circ}$ at $5^{\circ}, 3.81^{\circ}$ at $10^{\circ}$, and $6.83^{\circ}$ at $15^{\circ}$ eccentricity. A one-way ANOVA accordingly revealed a significant effect of eccentricity, $(F[1.23,11.07]=146.20, P<.0001$, GreenhouseGeisser corrected).

\title{
Typically Developing and Amblyopic Fovea
}

Acuity values for children with typical vision and amblyopia are plotted in Figure 3A. Gapsize thresholds for the left and right eyes of children with typical vision averaged 1.1 and 0.9 arcmins respectively (close to a Snellen acuity of 6/6). There was no significant difference between these values (paired samples t-test: $\mathrm{t}[19]=2.37, P=.5$ ), indicating no interocular differences in acuity. Reduced acuity levels were evident in the amblyopic eye of the amblyopic group, with an average of 4 arcmins compared to 1.1 arcmin for the unaffected fellow fixating eye (equivalent to Snellen acuities of $6 / 24$ and 6/6). This interocular difference was significant (paired samples t-test: $\mathrm{t}[19]=4.13, P<.001)$, consistent with the characteristic acuity deficit in amblyopia. Acuity in the fellow eye did not differ from the acuity of the children with typical vision (unpaired t-test between unaffected eye and both eyes of children with typical vision: $[[58]=-1.06, P=.29$ ). 
A.

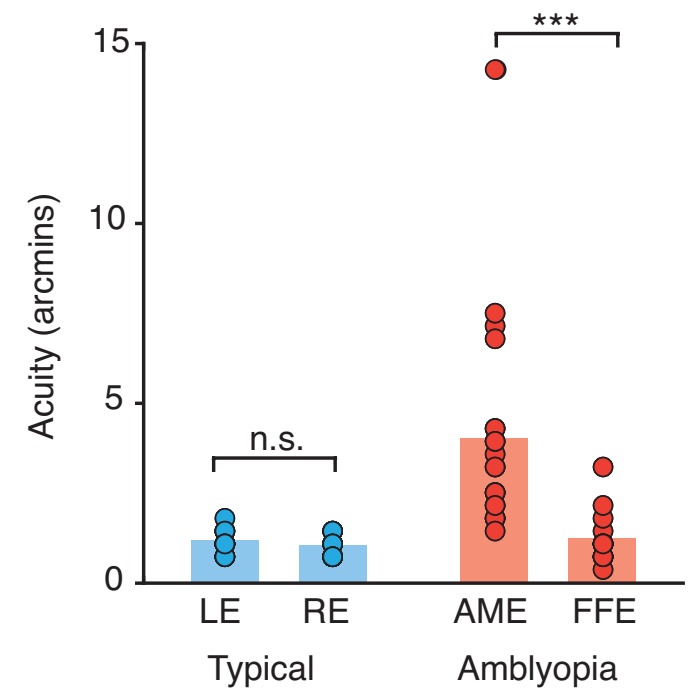

B.

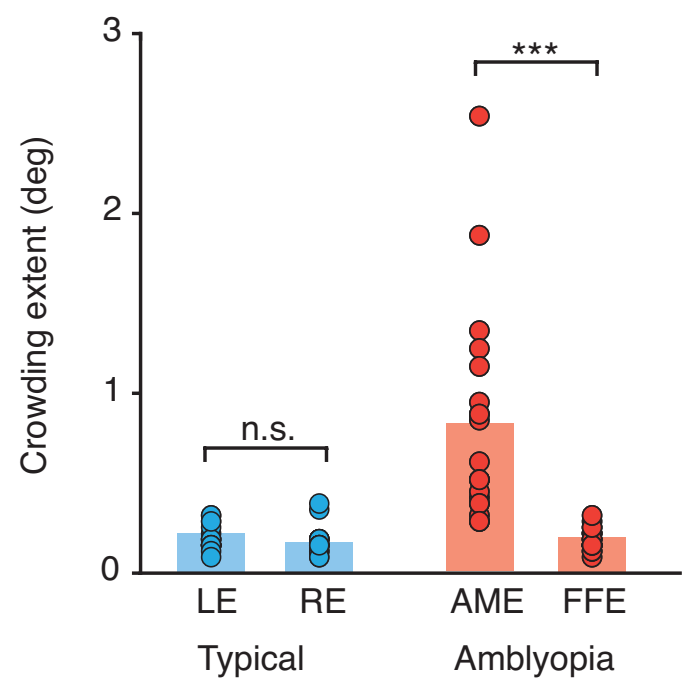

Figure 3. Acuity and crowding in the typically developing and amblyopic fovea.

A. Acuity measured as gap-size thresholds (in minutes of arc) for children with typical vision and amblyopia ( $\mathrm{n}=20$ each). Dots indicate values for each eye of individuals; bars indicate the mean. $L E=$ left eye, $R E=$ right eye, $A M E=$ amblyopic eye, $\mathrm{FFE}=$ fellow fixating eye, n.s. $=$ no significant difference, ${ }^{\star \star *} \mathrm{P}<.001$. B. The extent of crowding (in degrees of visual angle) measured as the centre-to-centre separation between the target and flankers, plotted as in panel $\mathrm{A}$.

Values for the spatial extent of crowding for children are plotted in Figure 3B. For children with typical vision, the extent of crowding averaged $0.17^{\circ}$ for the left eye and $0.15^{\circ}$ for the right eye, with no significant interocular difference (paired samples t-test: $\mathrm{t}[19]=1.58, P=.13$ ). For the amblyopic group, the extent of crowding was greater in the amblyopic eye, averaging $0.79^{\circ}$ compared to $0.16^{\circ}$ for the fellow fixating eye (paired samples t-test: $t[19]=485, P<.001$ ). There was no difference in the extent of crowding between the fellow fixating eye of the children with amblyopia and the two eyes of children with typical vision (unpaired t-test: $\mathrm{t}[58]=-0.18, P=.86$ ).

\section{Orientation-Matching Task}

Responses in the matching task were recorded as the perceived orientation of the target on each trial, which were subtracted from the veridical target value to give error values. Frequency histograms were constructed to tally the errors from $\pm 180^{\circ}$ in $10^{\circ}$ bins, separately for each flanker condition. For children, this gave five distributions of response errors per observer. For adults, responses were combined across the five repeat blocks collected to give five distributions for each eccentricity. Because the pattern of errors in conditions with equivalent target-flanker differences of opposite sign $\left(-30^{\circ}\right.$ and $30^{\circ},-90^{\circ}$ and $\left.90^{\circ}\right)$ were mirror-symmetric, the sign of the response errors was reversed in conditions with negative differences in order to sum the distributions. This gave three response-error distributions per observer (and per eccentricity for adults): unflanked, $30^{\circ}$ target-flanker difference, and $90^{\circ}$ target-flanker difference. 


\section{Adult Periphery}

Figure 4 plots response-error histograms for each eccentricity in the adult periphery. For unflanked targets (left column), the distribution of response errors was unimodal across all eccentricities (panels A-D), with a peak at $0^{\circ}$ and a narrow width. As such, when the target was presented in isolation, observers reported its orientation with good accuracy and precision, with increasing eccentricity having no effect on these estimates.

With flankers that differed by $30^{\circ}$ (middle column), response-error distributions were also unimodal at all four eccentricities, though with a peak at orientations between the target and flanker values (shown as dashed lines). In other words, the distribution of response errors shifted towards the flankers. There was also an increase in the spread of response errors relative to the unflanked condition. In other words, crowding had a disruptive effect on both accuracy and response precision.

When flankers differed by $90^{\circ}$ from the target, response-error distributions became bimodal (Figure 4, right column). The first peak was concentrated at $0^{\circ}$, indicating responses near to the veridical target value. The second peak was centred at $90^{\circ}$, near to the flanker orientation. The location of these peaks did not change with eccentricity, though the height of the peaks did. At lower eccentricities, the frequency of responses near the target was greater (i.e. the peak centred on $0^{\circ}$ was highest), whereas at larger eccentricities responses near the flanker orientation became more frequent. In other words, observers were increasingly likely to report orientations near to the flankers as eccentricity increased.

These distributions of response error allow us to draw a number of conclusions regarding the perceptual effects of crowding in the adult periphery. Observers were both accurate and precise when reporting the orientation of unflanked targets. With $\pm 30^{\circ}$ target-flanker differences, crowding primarily led observers to indicate intermediate orientations between the target and flankers. These responses can be classified as assimilation errors. Crowding with $\pm 90^{\circ}$ target-flanker differences led to a mixture of responses near to either the target or flanker orientations. The latter can be classified as substitution errors, which increased in frequency with eccentricity.

\section{Typically Developing and Amblyopic Fovea}

Figure 5 shows histograms of the response errors for children. When the target was unflanked, children with typical vision (Figure 5A, left) gave a unimodal distribution of response errors centred near $0^{\circ}$, with a slightly broader width than that found with adults in the periphery (Figure 4). The group with amblyopia (Figure 5B, left) showed an almost identical pattern of unflanked response errors as children with typical vision when stimuli were presented in the fovea of their amblyopic eye, with the peak centred on $0^{\circ}$ and a similar bandwidth. In other words, both groups could accurately report the orientation of the isolated target element with good precision. 


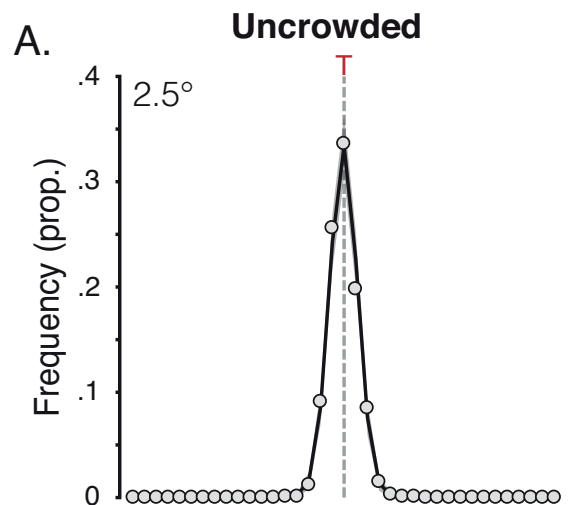

B.

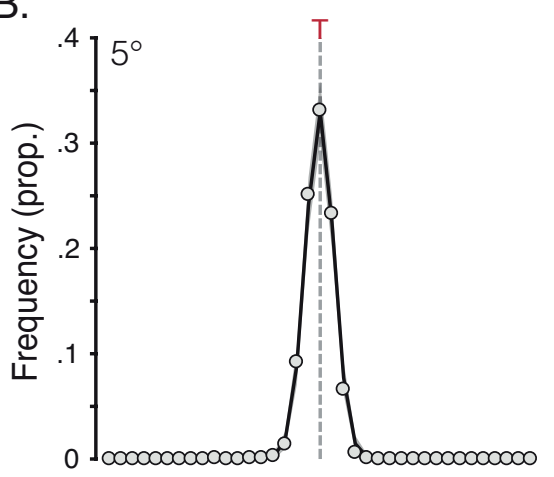

C.
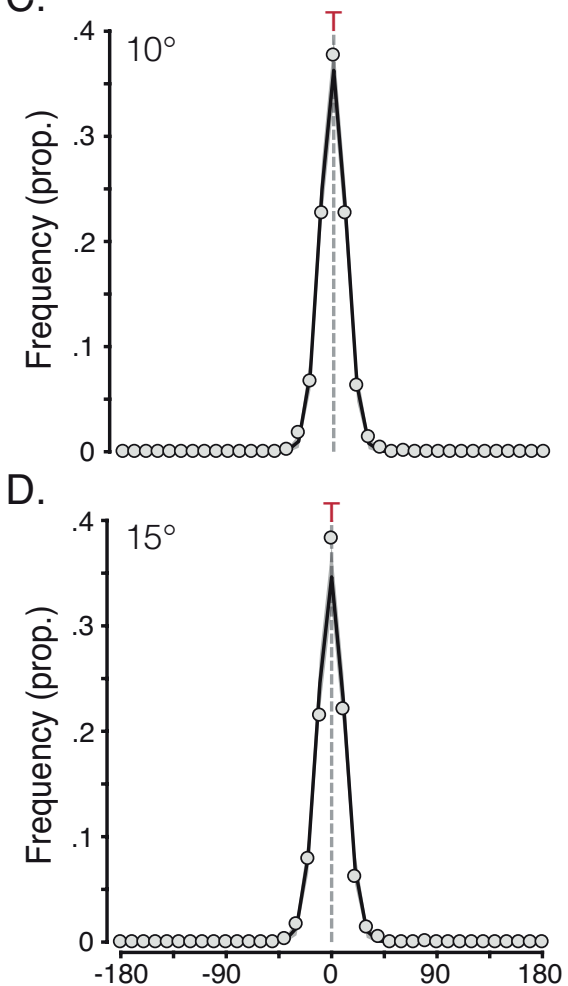

$30^{\circ}$ difference
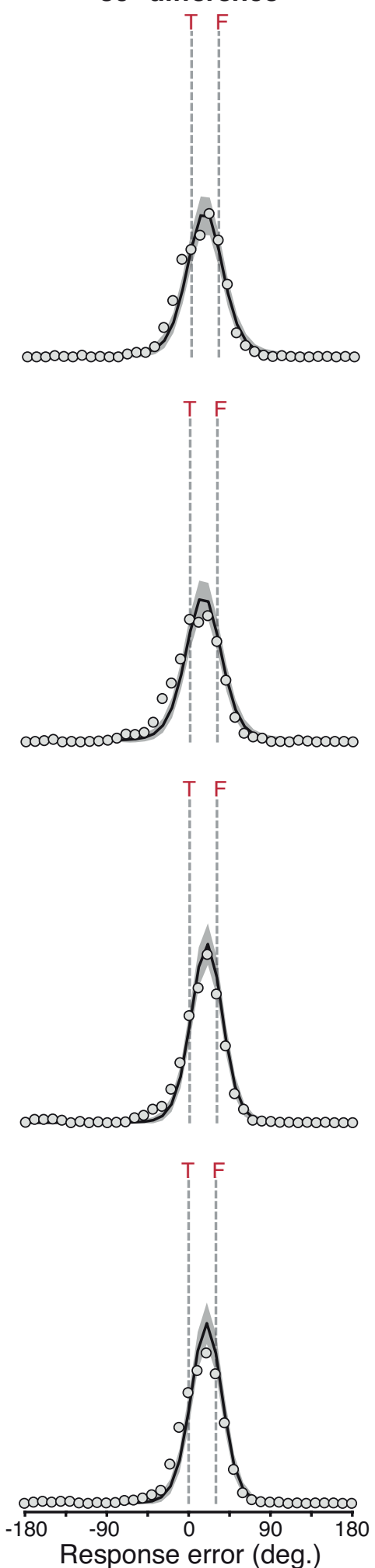
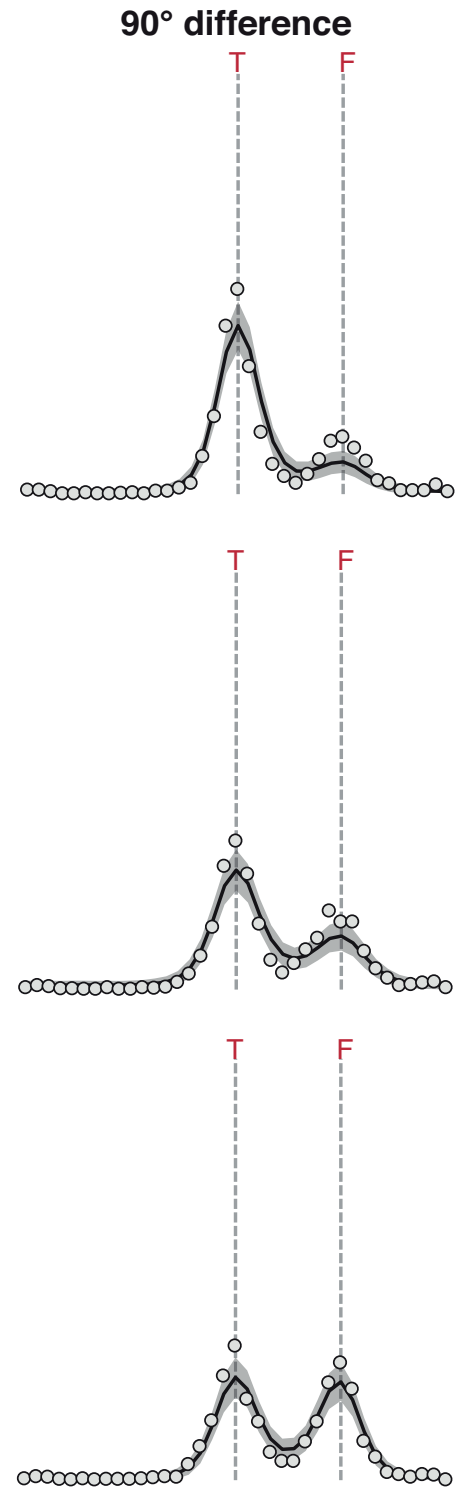

Figure 4. Distributions of mean response error from the orientation-matching task for the adult periphery.

A. Response error distributions at $2.5^{\circ}$ eccentricity, with mean values presented as light-grey dots. The black solid line plots the mean distribution of the population pooling model, with grey shaded areas plotting the $95 \%$ range of simulated distributions for 1000 model iterations. Dashed grey lines indicate the target location ('T'), and for the conditions in which flankers were present, the flanker location ('F'). B-D. Response error distributions at $5^{\circ}-15^{\circ}$ eccentricity, plotted as in panel A. 
bioRxiv preprint doi: https://doi.org/10.1101/2021.11.30.470647; this version posted December 2, 2021. The copyright holder for this preprint (which was not certified by peer review) is the author/funder, who has granted bioRxiv a license to display the preprint in perpetuity. It is made available under aCC-BY-NC-ND 4.0 International license.

When flankers differed from the target by $30^{\circ}$, both groups of children showed unimodal response-error distributions with a peak shifted towards the flanker orientation and centred near $15^{\circ}$. Both groups showed an increase in response-error variability compared to the unflanked condition, though this variability was greater in the amblyopic group. In other words, children from both groups primarily reported intermediate orientations between the target and flankers, with reduced response precision relative to unflanked performance, similar to that observed in the adult periphery.

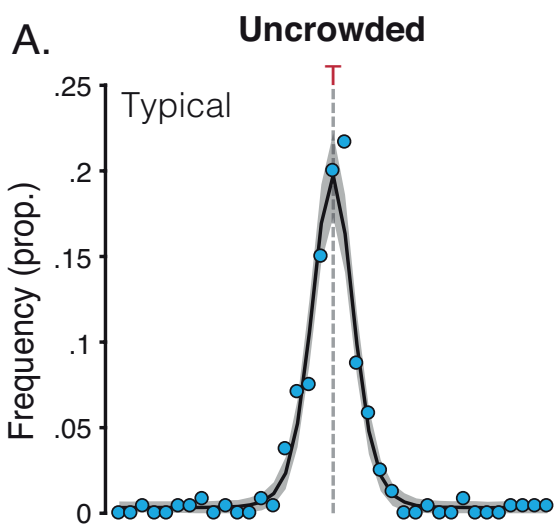

B.

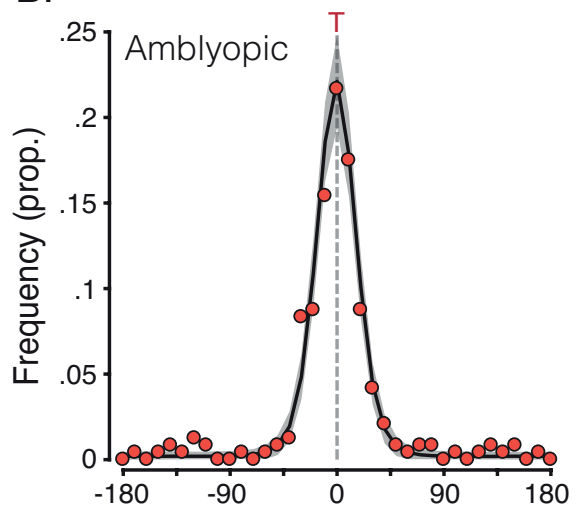

$30^{\circ}$ difference
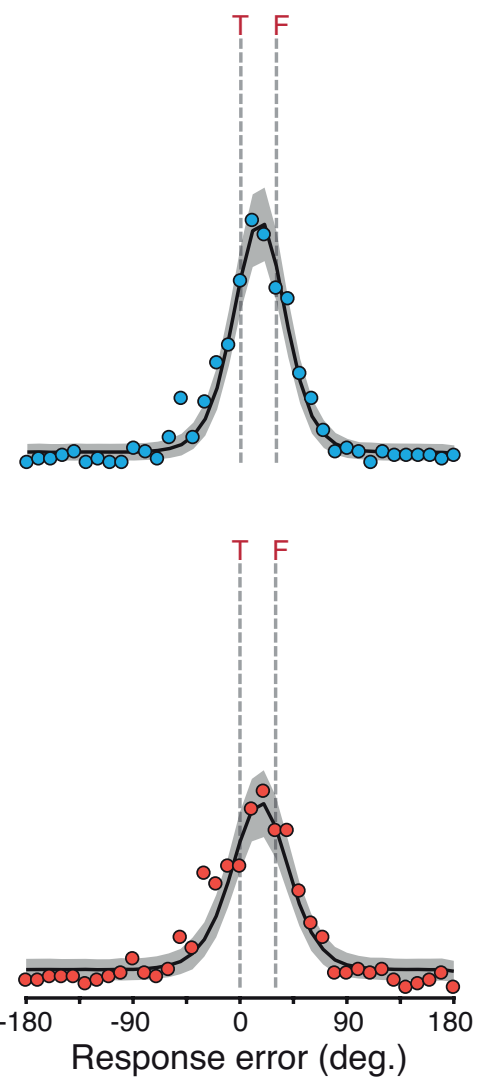

$90^{\circ}$ difference
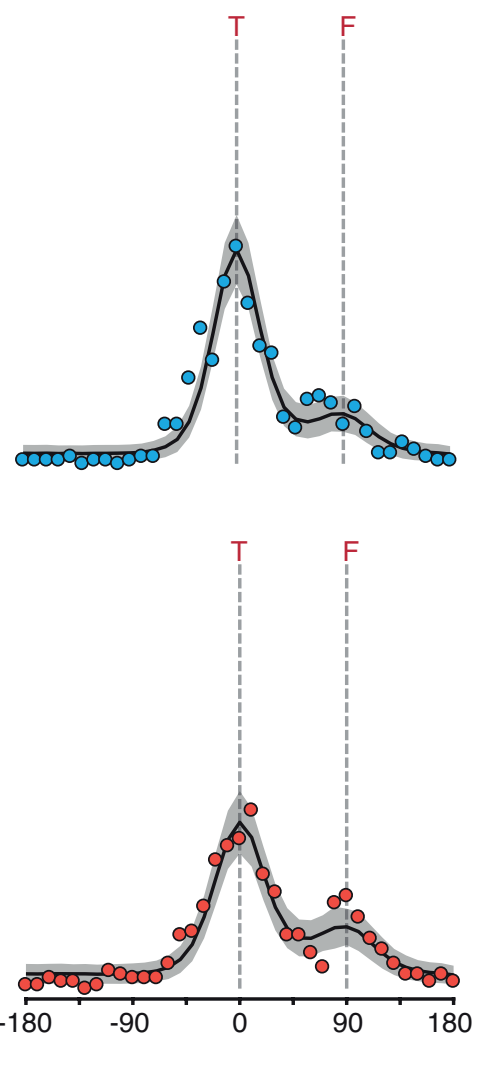

Figure 5. Mean response-error distributions for children with typical vision and amblyopia in the orientation-matching task.

A. Response-error distributions for children with typical vision, with mean values shown as dots. The black solid line indicates the mean distribution of the population pooling model, with grey shaded areas plotting the range of simulated distributions for 1000 model iterations. Dashed grey lines indicate the target orientation (T), and when present, the flankers (F). B. Response-error distributions for children with amblyopia, plotted as in panel A. Note that all data here is for the amblyopic eye.

When flankers differed by $90^{\circ}$, response-error distributions for both groups of children became bimodal. In each case, responses were most frequently near to the target orientation, with a secondary peak in responses close to $90^{\circ}$ error, indicative of flanker reports. These flanker responses were more frequent in children with amblyopia. There was also an increase in the variability of responses relative to unflanked performance, but to a lesser extent than when the 
flankers differed by $30^{\circ}$. These response-error distributions were highly similar to those of adults in the near periphery (Figure $4 \mathrm{A \& B}$ ).

Taken together, children performed well when required to judge the orientation of uncrowded targets, giving responses that were both accurate and precise. With $\pm 30^{\circ}$ target-flanker differences, errors were primarily reports of intermediate orientations between the target and flankers. As with adults in this condition, these can be classified as assimilation errors. With $\pm 90^{\circ}$ target-flanker differences, children primarily reported values near to the target orientation, with a secondary rate of responses to the flanker orientation. The latter can be classified as substitution errors, similar to the pattern of adult errors at closer eccentricities. Therefore, on a group level, children with typical vision and amblyopia made the same systematic errors as adults in the peripheral visual field.

\section{Modelling}

Given the common pattern of crowded errors made by adults and children with both typical vision and amblyopia, we next sought to examine the basis of these systematic perceptual outcomes with a computational model. Our approach was inspired by the models of van den Berg, Roerdink, and Cornelissen (2010) and Harrison and Bex (2015), and similar to recent models of crowding for colour and motion (Greenwood \& Parsons, 2020). The model had three distinct stages, summarised in Figure 6.

Panel A shows example stimuli for each flanker condition. For the ease of modelling, response errors were directly simulated by using the orientation difference from the target as inputs (rather than absolute orientations). As in the results presented above, $0^{\circ}$ indicated no error. In the first stage of the model (Figure 6B), the response of a population of detectors selective for orientation were simulated, following the well-documented orientation selectivity of neurons in primary visual cortex (Schiller, Finlay, \& Volman, 1976). Each detector responded to a range of orientations, according to a Gaussian tuning function with a peak sensitivity centred on a particular orientation, as in equation 1:

$$
r(\theta)=\alpha e^{\frac{(-\theta-\mu)^{2}}{2 \sigma^{2}}}+\gamma n
$$

Here, $r(\theta)$ is the population response at a given orientation $(\theta)$, ranging from $\pm 180^{\circ}$. The value $\alpha$ set the height of the detector sensitivity (set to 1), $\mu$ set the orientation producing the peak response, and $\sigma$ represented the bandwidth, set to $30^{\circ}$ to match the selectivity of neurons in cortical area V1 (De Valois, Yund, \& Hepler, 1982). Gaussian noise $n$ was added with a magnitude of $\gamma$ (the first free parameter). This 'early noise' allowed us to fit sensitivity to unflanked orientations in particular. 
bioRxiv preprint doi: https://doi.org/10.1101/2021.11.30.470647; this version posted December $2,2021$. The copyright holder for this preprint (which was not certified by peer review) is the author/funder, who has granted bioRxiv a license to display the preprint in perpetuity. It is made available under aCC-BY-NC-ND 4.0 International license.

Based on the principles of population coding (Pouget, Dayan, \& Zemel, 2000), the resulting population-response distribution is a Gaussian function centred on the orientation of the Landolt-C stimulus, with a bandwidth equivalent to the underlying sensitivity bandwidth of the detectors. On trials when the input to the model was a target presented in isolation (unflanked), the resulting population responses were centred near $0^{\circ}$. Responses from an example unflanked trial are shown in Figure 6B (top panel). For illustration, in this example trial the early noise is set to 0.1 .

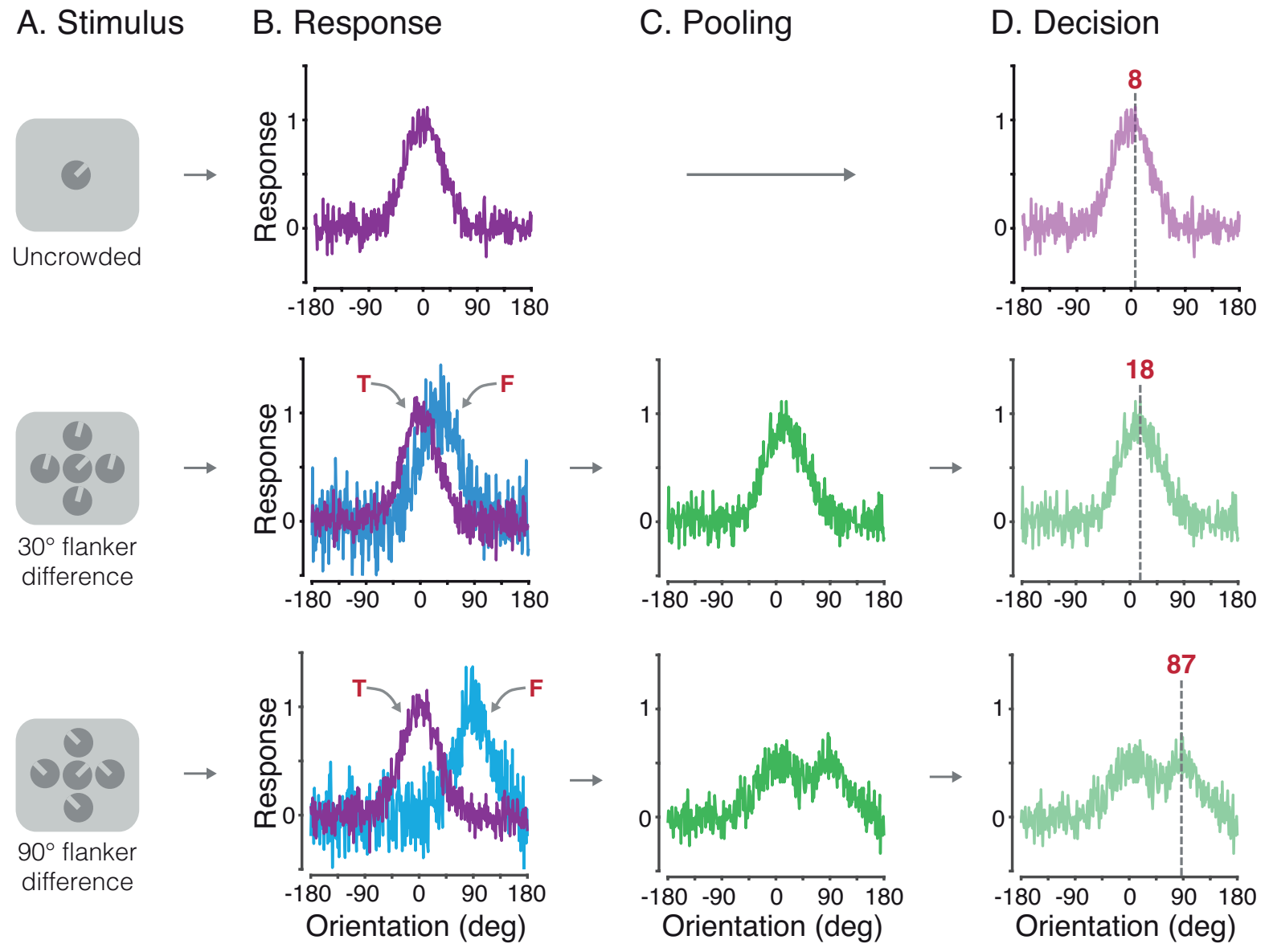

Figure 6. Illustration of the 3 stages of the weighted population pooling model.

A. Example stimulus input for each of the 3 flanker conditions (unflanked, and flankers with a $30^{\circ}$ or $90^{\circ}$ difference from the target). B. Early population response to the target (upper panel), and the target and flankers (middle and bottom panels). Arrows indicate the response to the target orientation (' $T$ '; purple distributions) and the response to the orientation of the flankers ('F'; blue distributions). C. The pooling stage, modelled as the weighted combination of population responses to the target and flankers. $\mathbf{D}$. The decision stage, where the perceptual outcome is read-out as the peak of the combined population response. The grey dashed line indicates this peak, with the decision value indicated numerically.

In the $30^{\circ}$ and $90^{\circ}$ flanker difference conditions, population responses were generated for both the target $\theta_{t}$ and the flanker orientation $\theta_{f}$ on each trial. Flanker responses were generated using equation 1, though with a second free parameter for the noise term ('late noise'), which allowed us to determine the degree of performance impaired induced by the flankers. The resulting 
population responses to the flanker orientation were centred either near $30^{\circ}$ or $90^{\circ}$, respectively.

Figure 6B shows population responses from an example trial for each of the two flanker conditions (middle and bottom rows), with the late noise parameter set to 0.2.

The second stage of the model simulated the effects of crowding. Here we followed recent models that depict crowding as a pooling process resulting from the combination of population responses to the target and flanker elements (van den Berg, Roerdink, \& Cornelissen, 2010; Harrison \& Bex, 2015; Greenwood \& Parsons, 2020). A weighted sum of the population response to the target and flankers was taken that allowed modulation of the precise combination of these population responses. The weighted combination of responses to the target and flankers was given as:

$$
y_{c}=\left(y_{t} w_{t}+y_{f} w_{f}\right)
$$

Here, $w_{t}$ and $w_{f}$ were the weights for the population responses to the target and flankers, respectively. The flanker weight ranged from $0-1$, with the weight of the target being one minus the flanker weight. As the data from all groups indicated that response-error distributions in the $30^{\circ}$ flanker difference condition differed from those in the $90^{\circ}$ flanker difference condition, the flanker weight was independent in these conditions. This gave two additional free parameters: the flanker weight for the $30^{\circ}$ condition $\left(w_{f_{30}}\right)$ and that for the $90^{\circ}$ condition $\left(w_{f_{90}}\right)$, to give four free parameters in total.

Figure $6 \mathrm{C}$ shows the second stage for each flanker condition on an example trial. With a $30^{\circ}$ target-flanker difference and a flanker weight of 0.5 , both target and flanker distributions contribute equally to the pooled response. Their breadth means that the combined population response distribution is broadly unimodal, with a peak that shifts away from $0^{\circ}$ towards the $30^{\circ}$ flanker orientation. With a $90^{\circ}$ target-flanker difference and the same 0.5 flanker weight, the combined population response distribution becomes bimodal, with peaks near the target and flanker orientations.

In the third and final stage of the model, a "decision" on the perceived target orientation was made by extracting the peak population response on each trial. For unflanked targets (Figure $6 \mathrm{D})$, the population response to the target carried through to the final stage, and the peak of the response is near the target at $8^{\circ}$. For the $30^{\circ}$ and $90^{\circ}$ flanker difference conditions, the peak of the combined target and flanker population responses was taken. In the $30^{\circ}$ flanker difference condition, this example trial results in a shift in the peak towards the flankers (at $\left.18^{\circ}\right)-$ an assimilation error. A decrease in the flanker weight here would shift the peak back towards the target orientation $\left(0^{\circ}\right)$, and vice versa. In the $90^{\circ}$ flanker condition, the combination of the bimodal 
distribution and noise results in a peak closer to the flankers (at $87^{\circ}$ ) - a flanker substitution error. A decrease in the flanker weight here would increase the likelihood of responses laying around the $0^{\circ}$ target value, rather than around the $90^{\circ}$ flanker.

Our model simulation included 1000 trials per flanker condition. The best-fitting parameters for the group of children with typical vision and amblyopia, and adults were determined using a two-stage fitting procedure. The initial coarse fit involved a grid search through the parameter space in pre-defined steps. From this we derived the parameters that best fit the data in the grid using the least-squared error (LSE) between response-error distributions and the simulated distributions from 1000 trials. In the second-stage fine fit, the best parameters from the coarse fit were used to seed the analysis, with the best-fitting parameters determined by minimising the LSE using fminsearch in Matlab, again taking the difference between data and simulated distributions from 1000 trials. We then ran 1000 iterations of the model with the best-fitting parameters for each dataset.

We also considered the response errors that would arise if crowding does not have a systematic effect on target appearance, but rather distorts or adds noise to the target orientation. To do so, we tested an additional model that did not contain the second pooling stage, but rather simply added noise to the population response when flankers were present. The noise model had three stages, the first of which was identical to the population pooling model, using equation 1 above to produce a population response to the target. Early noise was again the first free parameter. Population responses to the flankers were not simulated. Rather, the second stage differed in that the population response to the target was subjected to an additional noise parameter, of the form:

$$
y_{c}=y_{t}+\beta \sigma_{\eta}
$$

Here, $y_{t}$ is the population response to the target, $\sigma_{\eta}$ the added random noise and $\beta$ was the magnitude of this noise (the second and final free parameter in the noise model). The third stage of the model was then identical to the final stage of the pooling model, with the peak response taken as the 'decision' of the model on each trial. As with the population pooling model, we ran 1000 trials of this 3-stage model for each condition, using the procedure described above.

\section{Model Simulations of Group Data}

Adults

Figure 4 shows the result of 1000 iterations of the best-fitting population pooling model for the adult mean data at each eccentricity. For unflanked targets, the model almost perfectly captures the response errors, with early noise values that were similar across all eccentricities: 
0.30 at $2.5^{\circ}, 0.32$ at $5^{\circ}$ eccentricity, 0.26 at $10^{\circ}$ and 0.29 at $15^{\circ}$ eccentricity. These best-fitting parameters are plotted in Figure 7 (white triangles). When the target was crowded, response distributions became noisier and more broadly distributed, with a lower peak response. The model captured this through the addition of crowding noise to the flanker population response, which added further disruption to the pooling stage. Values for the crowding noise parameter showed a slight decrease with eccentricity, with values of 1.22 at $2.5^{\circ}, 1.19$ at $5^{\circ}, 0.76$ at $10^{\circ}$ and 0.73 at $15^{\circ}$ eccentricity.

The systematic nature of the response errors induced by flankers were driven by the flanker weight parameters. With $\pm 30^{\circ}$ target-flanker differences, at $2.5^{\circ}$ eccentricity the weight was 0.52 , which clearly replicates the response-error distribution in Figure 4A (middle) where the majority of the errors were between the target and flanker orientations. These flanker weights increased with eccentricity with 0.51 for $5^{\circ}$ eccentricity, 0.62 at $10^{\circ}$ and 0.64 at $15^{\circ}$. With $\pm 90^{\circ}$ target-flanker differences, there was a marked effect of eccentricity, with the proportion of flanker responses increasing with eccentricity. This was captured well by the model, which followed this pattern of increasing flanker responses with an increase in the flanker weights. These values increased from 0.34 at $2.5^{\circ}$ to 0.42 at $5^{\circ}, 0.50$ at $10^{\circ}$, and 0.52 at $15^{\circ}$ eccentricity. On the whole, the model provides an accurate characterisation of the profile of response errors in all conditions.

In contrast, the noise model generally performed poorly, as shown in Figure A1 (panels A-B) of Appendix B. Although the model was able to capture errors around the target in the unflanked condition, it failed to produce the shift towards the flankers in the $30^{\circ}$ condition and the bimodal pattern of errors in the $90^{\circ}$ condition. Clearly the addition of noise alone, ignoring the identity of the flankers, is insufficient to account for these errors. To determine which of the two models best fit the response distributions, we computed Akaike Information Criterion (AIC) values (Akaike, 1974) which take the least squared error (LSE), and correct for the number of parameters included in each model. This is important since the population pooling model had four free parameters, whereas the noise model had two. Despite this correction, the superior performance of the population pooling model is clear from the AIC values, shown in Figure 8A, where lower AIC values indicate better model fits to the data. In adults, the pooling model outperformed the noise model at all eccentricities. As such, the group response errors could not be accounted for by a process that merely adds random noise to the target orientation.

\section{Children}

Figure 5 shows the simulated distributions for the group data of children with typical vision (Figure 5A) and amblyopia (Figure 5B), each computed as the mean of 1000 model iterations. In the unflanked condition (Figure 5A \& B, left), the model captures the Gaussian distribution of response errors well for both groups, with early noise values of 0.78 for the children with typical vision and 
0.62 for the amblyopic group, both higher than best-fitting values for the group data of adults. When the target was crowded, the combined population response again became noisier and more broadly distributed, with a lower peak response. This was again captured by the model with the addition of crowding noise, with values of 1.40 for the group with typical vision and 1.84 for the group with amblyopia, both again higher than the values used for adults.

For the crowding condition with $\pm 30^{\circ}$ target-flanker differences, response error distributions were centred between $0^{\circ}$ and $30^{\circ}$. To reproduce this shift, flanker weights in the model were similar to those of the adult periphery, with 0.53 for the group of children with typical vision and 0.57 for the amblyopic group, similar to that seen with the two closest eccentricities in the adults. With $\pm 90^{\circ}$ target-flanker differences, the model successfully captured both peaks of the bimodal distribution of response errors in each of the two groups. The flanker weight for this condition was 0.36 for both the children with typical vision and the amblyopic group, again similar to adult values in the parafovea. Overall, the model successfully captured the pattern of systematic errors observed for both groups of children. The noise model again provided a substantially poorer fit to the data (see Appendix B and panels C-D of Figure A1), demonstrating that the errors of children with typical and amblyopic vision cannot be accounted for by a process that merely adds random noise to the target orientation.

\section{Model Simulations of Individual Data}

Having demonstrated that the population pooling model can successfully reproduce response-error distributions in the adult periphery, the developing fovea and the amblyopic fovea using data averaged across individuals, we next consider how well the model can account for individual data. The model was fit using the same procedure as above, this time to data from individual observers. Because individual children had so few trials per condition (24 in each crowded condition), smoothing was applied to the response-error histograms using a three-point boxcar average prior to model fitting. Figure 7 shows the best-fitting values for all free parameters of the model for each adult at the four eccentricities tested, and for each child in the groups with typical vision and amblyopia.

For the early noise parameter (Figure 7A), values were again similar across the four eccentricities tested in adults, with a range of values between 0.32 and 0.66 and mean values (shown as bars) that ranged from 0.44-0.47. These values were generally larger in children with typical vision and amblyopia, ranging from $0.40-2.54$, with means of 0.80 and 0.92 for those with typical vision and amblyopia, respectively. In other words, some children required similar noise values to adults in order to simulate their unflanked performance, though the majority were noisier. For the crowding noise parameter (Figure 7B), adult values again showed a slight decrease with eccentricity on average, with means of 1.58 at $2.5^{\circ}, 1.21$ at $5^{\circ}, 1.01$ at $10^{\circ}$, and 1.12 at $15^{\circ}$. 
Variability in individual parameters was greater here, with values ranging from 0.41-3.04. Children in both groups again had a greater range of crowding noise values, varying from $0.41-3.73$ overall, with means of 1.62 and 2.13 for the typical vision and amblyopic groups, respectively. Here the amblyopic children generally required more noise to match their responses than any other group, though many children were similar to adults in this parameter.

A.

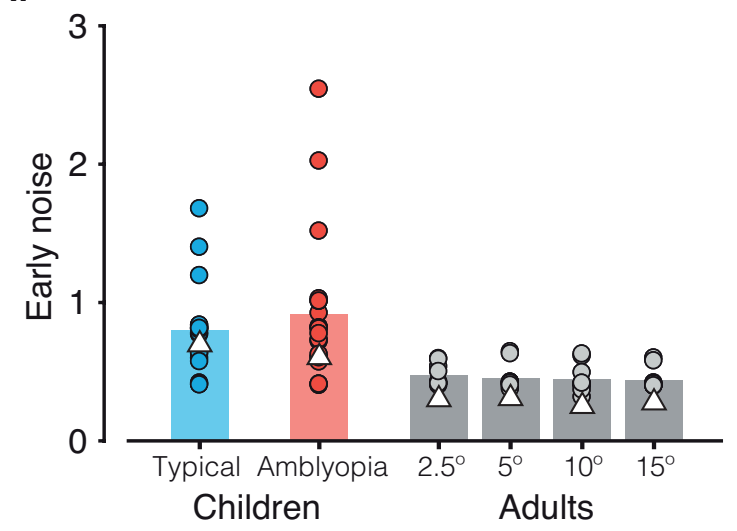

C.

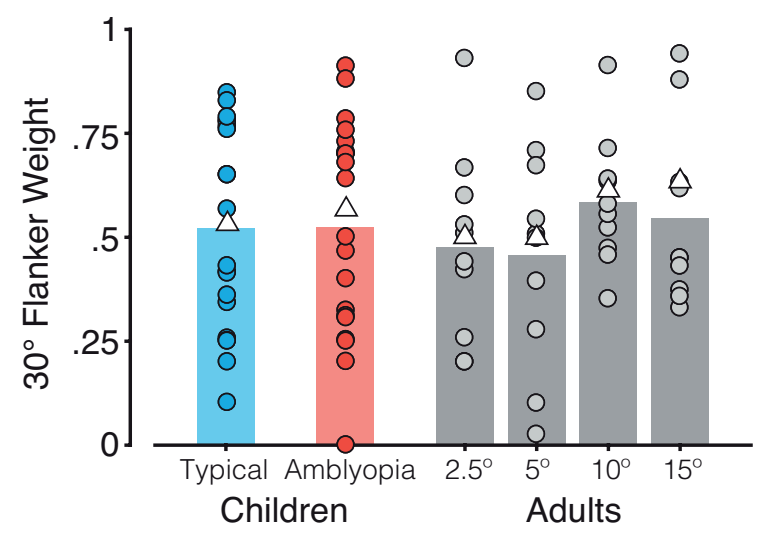

B.

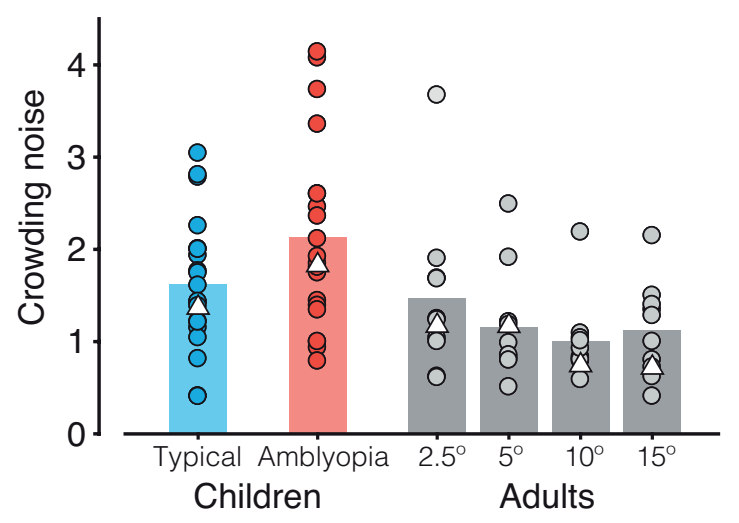

D.

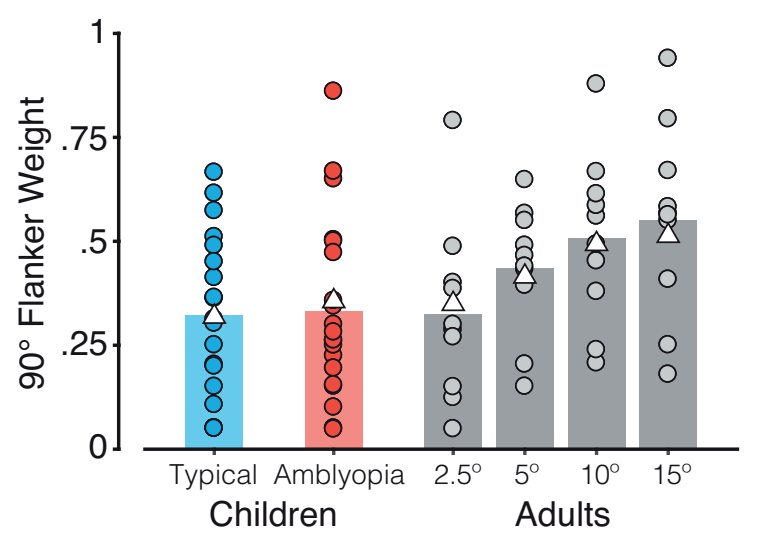

Figure 7. Best-fitting model parameters in the adult periphery and for children with typical vision and amblyopia.

A. Best-fitting values for the early noise parameter. Dots indicate individual observers, and bars the mean of the individual observers. White triangles show the parameters for fits to the averaged group data. B. Best-fitting values for the late noise parameter, plotted with conventions as in A. C-D. Best-fitting values for the flanker weights when the flankers differed by $30^{\circ}$ from the target (C), and when flankers differed by $90^{\circ}(\mathrm{D})$.

When flankers differed by $\pm 30^{\circ}$ from the target (Figure 7C), flanker weights again increased with eccentricity for adults, with means of $0.47,0.45,0.58$ and 0.55 from $2.5-15^{\circ}$ eccentricity. Substantial variations are also apparent, however, with values ranging from 0-0.94 across individuals at all eccentricities. For children with typical vision and amblyopia, the distribution of best-fitting flanker weights was similarly broad, varying from 0-0.91. Mean values were 0.52 and 0.53 , respectively, again corresponding to values in the adult periphery that were higher than the near periphery but lower than values at the farthest eccentricities. When flankers differed by $\pm 90^{\circ}$ from the target (Figure 7D), flanker weights in the adult periphery showed a strong effect of 
eccentricity, with mean best-fitting flanker weights gradually increasing from 0.32 at $2.5^{\circ}$ to 0.43 at $5^{\circ}, 0.51$ at $10^{\circ}$, and 0.55 at $15^{\circ}$. The range was again broad, however, covering $0.05-0.94$. In children, the range of flanker weight values was similarly broad, ranging from $0.05-0.86$, with means of 0.32 and 0.33 that were again most similar to the closer eccentricities in adults.

A number of conclusions can be drawn from the model fits to the individual data. First, children generally required larger early noise values than adults in order for the model to simulate their response error distributions. This indicates a broad difference the general properties of foveal vision in children and the adult periphery. Children from both groups also required greater crowding noise values than adults, suggesting greater difficulties in clutter that may go beyond the stimulus, perhaps into decisional processes. Flanker weights were however similar in the children to values for adults in the parafovea, particularly for the $90^{\circ}$ flanker condition, consistent with the commonalities observed in the pattern of response errors between adults and children.

In order to determine the success of these fits to individual data, the noise model was also fit to individual response-error distributions. Comparisons of these fits are shown in Figure A2 of Appendix $\mathrm{C}$ for adults and Figure A3 for children. Generally, as with the fits to the group data, the noise model failed to account for the systematic shift in the pattern of response errors towards the flanker orientations. However, for some individuals the noise model approached the success of the pooling model, and in others the noise model outperformed the pooling model.

AIC values for individual fits are shown in Figure 8B. In the adult periphery, the pooling model had lower AIC values than the noise model in 9 of 10 adults at $2.5^{\circ}$ and $5^{\circ}$, and for all 10 adults at $10^{\circ}$ and $15^{\circ}$. Accordingly, AIC values were significantly lower (indicating better fits) for the pooling model at all eccentricities: $\mathrm{t}[9]=-3.83, P=.004$ at $2.5^{\circ} ; \mathrm{t}[9]=-3.92, P=.004$ at $5^{\circ} ; \mathrm{t}[9]=-$ $7.35, P<.001$ at $10^{\circ}$ and $t[9]=-6.20, P<.001$ at $15^{\circ}$. Those for whom the pooling model failed tended to simply have low error rates (as shown in Figure A2), making it hard to discriminate between the models.

For children, all 20 of those with typical vision showed lower AIC values, indicating better fits for the pooling model, while 18 of 20 amblyopic children were better fit by the pooling model. Here too the AIC values were significantly lower (indicating better fits) for the pooling model, with $\mathrm{t}[19]=-5.96, P<.001$ for those with typical vision and $\mathrm{t}[19]=-3.67, P=.002$ for those with amblyopia. Amongst the amblyopic children, those for whom the pooling model failed tended to have highly noisy responses (as shown in Figure A3), which again gave similar fits for the two models. Although this tendency was present in some of the children with typical vision, this was never to the extent that the noise model outperformed the pooling model. 
A.

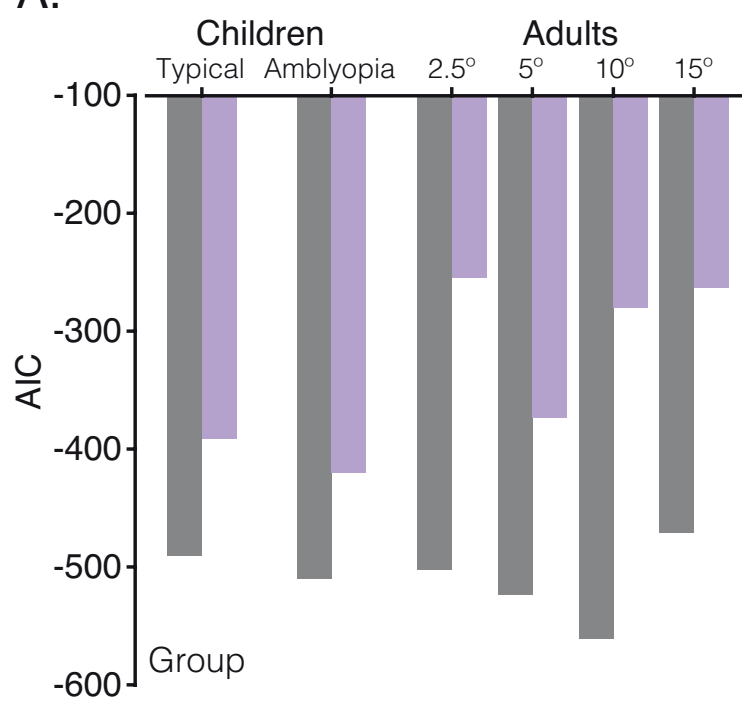

B.

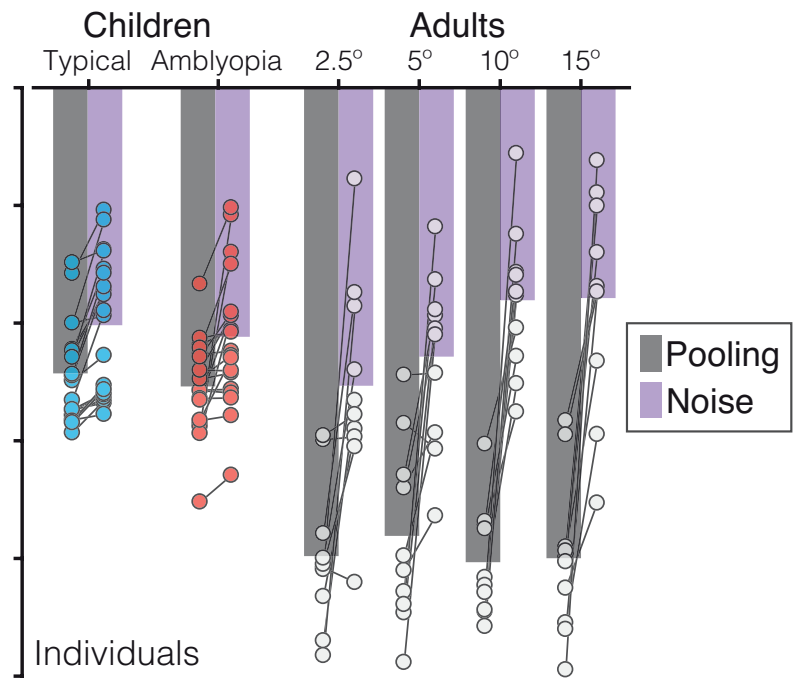

Figure 8. Akaike Information Criterion (AIC) values to assess goodness-of-fit for the pooling and noise models of crowding in each group.

A. AIC values for the fits to the averaged group data, plotted separately for the pooling (dark grey) and noise (light grey) models. Note that more negative values indicate better fits to the data. B. AIC values for model fits to individual data. Bars here represent the mean of the fits to individual data for the pooling and noise models, with individual values superimposed as circles. Individuals are joined with bars to show the direction of effect.

On the whole, the pooling model outperforms the noise model in describing the response errors of all three groups. It is particularly striking that the pooling model is able to outperform the noise model with fits to individual data, given that these distributions relied on only 24 trials for children in each crowded condition. We conclude that the errors made by adults in peripheral vision cannot be accounted for by a process that merely adds random noise to the target orientation. The same is true for children with both typical and amblyopic vision. Rather, systematic changes in the target orientation are required to capture the systematic errors in these patterns of performance.

\section{Discussion}

Our aim was to examine the perceptual effects of crowding in the fovea of children with typical vision and amblyopia and to assess its similarity with crowding in the typical adult periphery. In all three groups, errors in the perceived orientation of a crowded Landolt-C target were systematic in that they followed the appearance of the flanker elements. When the target was crowded, children made errors that predominantly matched either intermediate orientations between the target and flankers (assimilation) with $30^{\circ}$ target-flanker orientation differences, or the orientation of the flankers (substitution) with $90^{\circ}$ target-flanker differences (Figure 5). These errors matched those observed in adult peripheral vision (Figure 4), consistent with previous studies of adults (Parkes et al., 2001; Greenwood, Bex, \& Dakin, 2009; Dakin et al., 2010; Ester, Zilber, \& 
Serences, 2015; Harrison \& Bex, 2015). The frequency of both assimilation and substitution errors increased with eccentricity in the typical periphery, with the errors made by typical children and those with amblyopia most closely resembling the errors found at para-foveal eccentricities. We conclude that both children with typical vision and amblyopia make the same crowded errors as adults in the visual periphery, consistent with a common mechanism underlying crowding in these three instances.

We further demonstrate that a weighted population pooling model can reproduce this pattern of crowded errors in the typically developing and amblyopic fovea, as well as in the adult periphery. To do so, we followed population models of crowding (van den Berg, Roerdink, \& Cornelissen, 2010; Harrison \& Bex, 2015; Greenwood \& Parsons, 2020) in taking the perceived orientation of a crowded object from the response of a population of orientation-selective detectors. Crowding was produced by the weighted pooling of population responses to the target and flanker orientations. The weights determined the relative contribution of the target/flanker population responses to the pooled response distribution, from which decisions were drawn. With this approach, assimilation errors arose with $30^{\circ}$ target-flanker differences because the overlap in the two sets of population responses gave a peak response at intermediate orientations.

Substitution errors arose with $90^{\circ}$ target-flanker differences due to the bimodal distribution of the pooled response - although correct target responses were usually most common, noise in the bimodal pooled response gave a secondary rate of reports near the flanker orientation. Flanker population responses also added noise to the pooled population response, in line with previous models of peripheral crowding (Greenwood, Bex, \& Dakin, 2009; Ester, Klee, \& Awh, 2014; Ester, Zilber, \& Serences, 2015; Greenwood \& Parsons, 2020). However, noise alone was insufficient to account for the errors made by either group of children or in the adult periphery (Figure 8). The success of the population pooling model was apparent not only in the fits to group data, but also in the characterisation of individual distributions of response errors. Altogether, we suggest that pooling models provide a likely candidate for the common mechanism in these three instances of crowding.

More broadly, the population pooling model is consistent with two-stage theories of crowding (Levi, Hariharan, \& Klein, 2002b; Pelli, Palomares, \& Majaj, 2004; Levi, 2008). The initial feature-detection stage is here represented by the initial population responses in our model (separately for the target and flanker orientations). The subsequent feature-integration stage is then given by the weighted pooling of these population responses. The pooling process used herein is also consistent with higher-dimensional 'texture' pooling models, which simulate crowding through the extraction of image statistics across large regions of the peripheral field (Balas, Nakano, \& Rosenholtz, 2009; Freeman \& Simoncelli, 2011; Keshvari \& Rosenholtz, 2016; Rosenholtz, Yu, \& 
Keshvari, 2019). Both assimilation and substitution errors have been simulated with this approach in the adult periphery (Keshvari \& Rosenholtz, 2016). A similar process could work in the developing and amblyopic fovea, though it is unclear whether these models could also predict the increase in random responses found in children, as well as the individual differences observed.

The systematic effects of crowding that we observe are difficult to reconcile with higherlevel models of crowding. For instance, attentional models (Strasburger, Harvey, \& Rentschler, 1991; Strasburger, 2005) predict a predominance of substitution errors due to an inability to accurately focus spatial attention. The assimilation errors that we observe with $30^{\circ}$ target-flanker differences are difficult to explain within this framework. Similarly, grouping theories propose that crowding is determined by Gestalt principles in a top-down fashion (Herzog, Sayim, Chicherov, \& Manassi, 2015). These models fail to account for our data in two ways. First, they make no prediction regarding the systematic perceptual outcomes of crowding, focusing instead on the performance decrements induced by clutter. Second, their top-down operation is inconsistent with the monocular elevations in foveal crowding found in amblyopia - the same stimulus can cause crowding effects when presented to one eye, but not the other.

Our results also reveal substantial individual differences in crowding. Consistent with prior reports of individual differences in the spatial extent of crowding (Toet \& Levi, 1992; Petrov \& Meleshkevich, 2011; Greenwood, Szinte, Sayim, \& Cavanagh, 2017), here we observe variations in the propensity of individuals to make systematic errors in judgements of appearance, even for the same stimulus. This was true in adults, but also in children. For instance, with the $30^{\circ}$ target-flanker difference, some typically developing children reported clearly intermediate orientations, whereas others reported orientations more similar to the flankers (see Figure A3 and Appendix C). The pooling model was nonetheless able to account for these individual differences through variations in the four free parameters. Although children showed substantial variations in these parameters relative to adults, some of this variation may be due to differences in age, given the developmental trajectory of crowding (Jeon et al., 2010), as well as variations in the extent of disruption from amblyopia.

Given the consistency of the response-error distributions in these three instances, and the success of the population pooling model in reproducing them, we suggest that a common neural basis could underlie these effects. Although our model is agnostic with regards to the neural site of crowding, we suggest two possible factors that could drive the variations in flanker weights that modulate the strength of crowding. First, the increase in the extent of crowding with eccentricity (Bouma, 1970; Toet \& Levi, 1992) has been attributed to an insufficient sampling of the peripheral field, driven by increases in receptive field size (Parkes et al., 2001; Motter, 2009). Individual differences in crowding are indeed correlated with population receptive field (pRF) size in V2 (He, 
Wang, \& Fang, 2019), with increased pRF sizes observed in the amblyopic fovea in areas V1-V3 (Clavagnier, Dumoulin, \& Hess, 2015). The role of receptive field size in developmental crowding is less clear, with neuroimaging studies suggesting that pRFs reach adult-like sizes by 6 years of age (Dekker et al., 2019), despite foveal crowding being elevated until as late as 12 years (Atkinson \& Braddick, 1983; Atkinson et al., 1987; Jeon et al., 2010; Greenwood et al., 2012). It is possible however that the spatial selectivity of visual neurons may change in later childhood, given for instance the maturation of connections in primary visual cortex (Huttenlocher, de Courten, Garey, \& Van der Loos, 1982; Huttenlocher \& Dabholkar, 1997), and the later maturation of centresurround receptive fields in extrastriate cortex (Zhang et al., 2005).

Alternatively, the rise in crowding with eccentricity has also been attributed to decreases in the cortical distance between target and flanker elements (Motter \& Simoni, 2007; Mareschal, Morgan, \& Solomon, 2010) driven by cortical magnification (Daniel \& Whitteridge, 1961; Van Essen, Newsome, \& Maunsell, 1984; Sereno et al., 1995). Individual differences in cortical magnification across the visual field may then explain variations in crowding, similar to proposals regarding variations in acuity (Duncan \& Boynton, 2003) and perceived object size (Moutsiana et al., 2016). Amblyopia is known to produce a reduction in neurons responding to the amblyopic eye in areas V1 and V2 (Crawford \& von Noorden, 1979; Bi et al., 2011; Shooner et al., 2015), which could reduce cortical magnification and increase crowding. However, neuroimaging studies have thus far failed to observe differences in cortical magnification in either amblyopia (Clavagnier, Dumoulin, \& Hess, 2015) or in the course of typical development (Dekker et al., 2019). Although we cannot distinguish between the effects of receptive field size and cortical distance on the basis of our current data, the appealing prospect is that one or both of these factors might drive the variations in weights that determine the appearance of crowded objects in our model.

Variations in the featural selectivity of crowding are also evident in our results. Both children and adults (in the near periphery) gave more responses near to the correct target orientation when target and flankers differed by $\pm 90^{\circ}$ than when they differed by $\pm 30^{\circ}$. This finding is consistent with the selectivity of peripheral crowding for target-flanker similarity in orientation, whereby errors decrease when elements are less similar (Andriessen \& Bouma, 1976; Leat, Li, \& Epp, 1999; Levi, Hariharan, \& Klein, 2002b; Hariharan, Levi, \& Klein, 2005). Our findings reveal that target-flanker similarity also matters in developing and amblyopic crowding. This is inconsistent with prior reports that amblyopic crowding in adults disrupts target recognition regardless of target-flanker similarity (Hess, Dakin, Tewfik, \& Brown, 2001; Hariharan, Levi, \& Klein, 2005). Interestingly, at higher eccentricities in the adult periphery, we observe an increase in the frequency of substitution errors with $90^{\circ}$ target-flanker differences, suggesting that the selectivity for target-flanker similarity may decrease with eccentricity. Following suggestions that foveal crowding in amblyopia can be linked 
with an equivalent eccentricity in the typical periphery (Levi, Klein, \& Aitsebaomo, 1985), we note that the developing and amblyopic fovea resemble para-foveal eccentricities more than the far periphery in terms of both the selectivity for target-flanker similarity and the systematicity of crowded errors. It is possible that individual differences in crowding strength may then lead to differences in its selectivity for target-flanker similarity, given the apparent pairing of these properties.

The population pooling model reproduced this increase in the rate of substitution errors with eccentricity through changes in the weights applied to the target and flanker population responses. An alternative explanation for these errors is that they may arise due to increases in the positional uncertainty regarding the target element. Positional uncertainty could create source confusion (Wolford, 1975) either through the mislocalisation of features or whole letters (Strasburger, Rentschler, \& Jüttner, 2011). Indeed, both adult peripheral vision and amblyopic foveal vision are characterised by increased positional uncertainty (Hussain et al., 2015). Similarly, positional uncertainty may recede in the course of development, given the observation that Vernier acuity does not reach adult levels until the early teens (Carkeet, Levi, \& Manny, 1997; Skoczenski \& Norcia, 2002). As such, some proportion of the substitution errors found with $90^{\circ}$ target-flanker differences may be attributable to position uncertainty in the adult periphery, as well as in children with amblyopia and typical vision. Accordingly, the crowding noise parameter in our model was much higher in children than in adults, particularly for children with amblyopia, which could reflect an additional degree of source confusion. This is not necessarily inconsistent with pooling models of crowding, and indeed some have incorporated both processes (Freeman, Chakravarthi, \& Pelli, 2012; Harrison \& Bex, 2017), though it would speak against the idea raised above that a common factor could drive all of the observed variations, as some have suggested (Agaoglu \& Chung, 2016). Importantly however, position uncertainty alone cannot explain our results, given the predominance of assimilation errors observed with $30^{\circ}$ target-flanker differences.

Despite the above-noted similarities between groups, differences are also evident. The model fits to data from both sets of children required higher levels of early noise in order to match performance, consistent with the broader elevations in low-level vision such as acuity and contrast sensitivity, both in amblyopia (McKee, Levi, \& Movshon, 2003) and during development (Simons, 1983; Leat, Yadav, \& Irving, 2009). A small subset of children with amblyopia were further noted to have made a disproportionate amount of random reports (Figure A3), giving orientations that did not correspond to either the target or the flankers. For these cases, a model that simply adds noise to the target population response performed best (Figure 8). This increased response variability could be due to attentional lapses, or to perceptual distortions that characterise amblyopic vision (Pugh, 1958; Sireteanu, Lagreze, \& Constantinescu, 1993; Barrett et al., 2003). As with positional 
uncertainty however, distortions alone cannot account for the systematic effects of crowding on target appearance, though it is possible that they acted as an additional source of errors, particularly for some children.

We have presented a population pooling model that can characterise the perceptual effects of crowding in three instances: the amblyopic fovea, the typically developing fovea, and the adult periphery. On the basis of this common mechanism, we can make predictions for a diverse range of conditions where elevated crowding has been observed, including Posterior Cortical Atrophy (Crutch \& Warrington, 2007, 2009), dyslexia (Geiger \& Lettvin, 1987; Atkinson, 1991; Martelli et al., 2009), and infantile nystagmus (Chung \& Bedell, 1995; Pascal \& Abadi, 1995). Several of these conditions report similar properties to those observed herein. For instance, crowding in Posterior Cortical Atrophy exhibits a selectivity for target-flanker similarity that matches that observed in typical peripheral vision (Yong et al., 2014). In these instances, we would expect to see the same systematic pattern of errors as in the present study. In other cases, the properties of crowding appear to differ. For instance, the spatial extent of interference zones are elongated horizontally in idiopathic infantile nystagmus, unlike the radially symmetric zones observed in typical vision and the amblyopic fovea (Tailor et al., 2021). In these cases, the pattern of response errors may differ substantially. By examining the nature of the errors produced in these distinct conditions, we can thus determine the generality of this common mechanism for crowding.

\section{Acknowledgements}

This work was funded by the Moorfields Eye Charity (ST1411F) and the UK Medical Research Council (MR/K024817/1). Thanks to Dina Prapa from Moorfields Eye Hospital for her help with participant recruitment.

\section{References}

Agaoglu, M. N., \& Chung, S. T. L. (2016). Can (should) theories of crowding be unified? Journal of Vision, 16(15), 10.

Akaike, H. (1974). A new look at the statistical model identification. IEEE Transactions on Automatic Control, 19(6), 716-723.

Andriessen, J. J., \& Bouma, H. (1976). Eccentric vision: Adverse interactions between line segments. Vision Research, 16, 71-78.

Atkinson, J. (1991). Review of human visual development: Crowding and dyslexia. In J. F. Stein (Ed.), Vision and visual dysfunction (Vol. 13, pp. 44-57). London: Macmillan Press.

Atkinson, J., Anker, S., Evans, C., \& Mclntyre, A. (1987). The Cambridge Crowding Cards for preschool visual acuity testing. Paper presented at the Transactions of the 6th International Orthoptic Congress, Harrogate, UK.

Atkinson, J., \& Braddick, O. J. (1983). Assessment of visual acuity in infancy and early childhood. Acta Ophthalmologica Scandinavica, 157, 18-26.

Balas, B., Nakano, L., \& Rosenholtz, R. (2009). A summary-statistic representation in peripheral vision explains visual crowding. Journal of Vision, 9(12), 1-13. 
Barrett, B. T., Pacey, I. E., Bradley, A., Thibos, L. N., \& Morrill, P. (2003). Nonveridical visual perception in human amblyopia. Investigative Ophthalmology \& Visual Science, 44(4), 15551567.

Bi, H., Zhang, B., Tao, X., Harwerth, R. S., Smith, E. L., \& Chino, Y. M. (2011). Neuronal responses in visual area V2 (V2) of macaque monkeys with strabismic amblyopia. Cerebral Cortex, 21(9), 2033-2045.

Bouma, H. (1970). Interaction effects in parafoveal letter recognition. Nature, 226(241), 177-178.

Brainard, D. H. (1997). The Psychophysics Toolbox. Spatial Vision, 10(4), 433-436.

Carkeet, A., Levi, D. M., \& Manny, R. E. (1997). Development of Vernier acuity in childhood. Optometry and Vision Science, 74(9), 741-750.

Chung, S. T., Levi, D. M., \& Legge, G. E. (2001). Spatial frequency and contrast properties of crowding. Vision Research, 41(14), 1833 - 1850.

Chung, S. T. L., \& Bedell, H. E. (1995). Effect of retinal image motion on visual acuity and contour interaction in congenital nystagmus. Vision Research, 35(21), 3071-3082.

Clavagnier, S., Dumoulin, S. O., \& Hess, R. F. (2015). Is the cortical deficit in amblyopia due to reduced cortical magnification, loss of neural resolution, or neural disorganisation? The Journal of Neuroscience, 35(44), 14740-14755.

Coates, D. R., Levi, D. M., Touch, P., \& Sabesan, R. (2018). Foveal Crowding Resolved. Scientific Reports, 8(1), 9177.

Crawford, M. L., \& von Noorden, G. K. (1979). The effects of short-term experimental strabismus on the visual system in Macaca mulatta. Investigative Ophthalmology \& Visual Science, 18, 496-505.

Crutch, S. J., \& Warrington, E. K. (2007). Foveal crowding in posterior cortical atrophy: A specific early-visual-processing deficit affecting word reading. Cognitive Neuropsychology, 24(8), 843-866.

Crutch, S. J., \& Warrington, E. K. (2009). The relationship between visual crowding and letter confusability: Towards an understanding of dyslexia in posterior cortical atrophy. Cognitive Neuropsychology, 26(5), 471-498.

Dakin, S. C., Cass, J., Greenwood, J. A., \& Bex, J. P. (2010). Probabilistic, positional averaging predicts object-level crowding effects with letter-like stimuli. Journal of Vision, 10(10), 1-16.

Daniel, P. M., \& Whitteridge, D. (1961). The representation of the visual field on the cebral cortex in monkeys. Journal of Physiology, 159, 203-221.

Danilova, M. V., \& Bondarko, V. M. (2007). Foveal contour interaction and crowding effects at the resolution limit of the visual system. Journal of Vision, 7(2).

De Valois, R. L., Yund, E. W., \& Hepler, N. (1982). The orientation and direction selectivity of cells in macaque visual cortex. Vision Research, 22, 531-544.

Dekker, T. M., Schwarzkopf, D. S., de Haas, B., Nardini, M., \& Sereno, M. I. (2019). Population receptive field tuning properties of visual cortex during childhood. Developmental Cognitive Neuroscience, 37, 100614.

Duncan, R. O., \& Boynton, G. M. (2003). Cortical magnification within human primary visual cortex correlates with acuity thresholds. Neuron, 38, 659-671.

Ester, E. F., Klee, D., \& Awh, E. (2014). Visual crowding cannot be wholly explained by feature pooling. Journal of Experimental Psychology: Human Perception \& Performance, 40(3), 1022.

Ester, E. F., Zilber, E., \& Serences, J. T. (2015). Substitution and pooling in visual crowding induced by similar and dissimilar distractors. Journal of Vision, 15(1), 4-4.

Flom, M. C., Weymouth, F. W., \& Kahneman, D. (1963). Visual resolution and contour interaction. Journal of the Optical Society of America, 53(9), 1026-1032.

Freeman, J., Chakravarthi, R., \& Pelli, D. G. (2012). Substitution and pooling in crowding. Attention, Perception, \& Psychophysics, 74(2), 379-396.

Freeman, J., \& Simoncelli, E. (2011). Metamers of the ventral stream. Nature Neuroscience, 14 (9), 1195-1201. 
Geiger, G., \& Lettvin, J. Y. (1987). Peripheral vision in persons with dyslexia. New England Journal of Medicine, 316(20), 1238-1243.

Greenwood, J. A., Bex, P. J., \& Dakin, S. C. (2009). Positional averaging explains crowding with letter-like stimuli. Proceedings of the National Academy of Sciences of the United States of America, 106, 12130-13135.

Greenwood, J. A., Bex, P. J., \& Dakin, S. C. (2010). Crowding changes appearance. Current Biology, 20(6), 496-501.

Greenwood, J. A., \& Parsons, M. J. (2020). Dissociable effects of visual crowding on the perception of color and motion. Proceedings of the National Academy of Sciences of the United States of America, 117(14), 8196-8202.

Greenwood, J. A., Szinte, M., Sayim, B., \& Cavanagh, P. (2017). Variations in crowding, saccadic precision, and spatial localisation reveal the shared topology of spatial vision. Procedings of the National Academy of Sciences of the United States of America, 114(17), E3573- E3582.

Greenwood, J. A., Tailor, V. K., Sloper, J. J., Simmers, A. J., Bex, P. J., \& Dakin, S. C. (2012). Visual acuity, crowding and stereo-vision are linked in children with and without amblyopia. Investigative Ophthalmology \& Visual Science, 53(12), 7655-7665.

Hariharan, S., Levi, D. M., \& Klein, S. A. (2005). "Crowding" in normal and amblyopic vision assessed with Gaussian and Gabor C's. Vision Research, 45(5), 617-633.

Harrison, W. J., \& Bex, P. J. (2015). A unifying model of orientation crowding in peripheral vision. Current Biology, 25(24), 3213-3219.

Harrison, W. J., \& Bex, P. J. (2017). Visual crowding is a combination of an increase of positional uncertainty, source confusion, and featural averaging. Scientific Reports, 7, 45551.

He, D., Wang, Y., \& Fang, F. (2019). The critical role of V2 population receptive fields in visual orientation crowding. Current Biology, 29(13), 2229-2236. e2223.

Herzog, M. H., Sayim, B., Chicherov, V., \& Manassi, M. (2015). Crowding, grouping, and object recognition: A matter of appearance. Journal of Vision, 15, 1-18.

Hess, R. F., Dakin, S. C., Tewfik, M., \& Brown, B. (2001). Contour interaction in amblyopia: Scale selection. Vision Research, 41(17), 2285-2296.

Hussain, Z., Svensson, C.-M., Besle, J., Webb, B. S., Barrett, B. T., \& McGraw, P. (2015). Estimation of cortical magnification from positional error in normally sighted and amblyopic subjects. Journal of Vision, 15(2), 25.

Huttenlocher, P. R., \& Dabholkar, A. S. (1997). Regional differences in synaptogenesis in human cerebral cortex. The Journal of Comparative Neurology, 387(167-178).

Huttenlocher, P. R., de Courten, C., Garey, L. J., \& Van der Loos, H. (1982). Synaptogenesis in human visual cortex - Evidence for synapse elimination during normal development. Neuroscience Letters, 33(3), 247-252.

Jeon, S. T., Hamid, J., Mauer, D., \& Lewis, T. L. (2010). Developmental changes during childhood in single-letter acuity and its crowding by surrounding contours. Journal of Experimental Child Psychology, 107(4), 423-437.

Kalpadakis-Smith, A. V., Goffaux, V., \& Greenwood, J. A. (2018). Crowding for faces is determined by visual (not holistic) similarity: Evidence from judgements of eye position. Scientific Reports, 8(1), 12556.

Keshvari, S., \& Rosenholtz, R. (2016). Pooling of continuous features provides a unifying account of crowding. Journal of Vision, 16(3), 39.

Kooi, F. L., Toet, A., Tripathy, S. P., \& Levi, D. M. (1994). The effect of similarity and duration on spatial interaction in peripheral vision. Spatial Vision, 8, 255-279.

Krumhansl, C. L., \& Thomas, E. A. (1977). Effect of level of consfusability on reporting letters from briefly presented visual displays. Perception \& Psychophysics, 21, 269-279.

Leat, S. J., Li, W., \& Epp, K. (1999). Crowding in central and eccentric vision: The effects of contour interaction and attention. Investigative Ophthalmology \& Visual Science, 40, 504-512.

Leat, S. J., Yadav, N. K., \& Irving, E. L. (2009). Development of visual acuity and contrast sensitivity in children. Journal of Optometry, 2(1), 19-26. 
Levi, D. M. (2008). Crowding - An essential bottleneck for object recognition: A mini-review. Vision Research, 48(5), 635-654.

Levi, D. M., Hariharan, S., \& Klein, S. A. (2002a). Suppressive and facilitatory spatial interactions in amblyopic vision. Vision Research, 42, 1379-1394.

Levi, D. M., Hariharan, S., \& Klein, S. A. (2002b). Suppressive and facilitatory spatial interactions in peripheral vision: Peripheral crowding is neither size invariant nor simple contrast masking. Journal of Vision, 2, 3.

Levi, D. M., \& Klein, S. A. (1985). Vernier acuity, crowding and amblyopia. Vision Research, 25(7), 979-991.

Levi, D. M., Klein, S. A., \& Aitsebaomo, A. P. (1985). Vernier acuity, crowding and cortical magnification. 1985, 25(7), 963-977.

Levi, D. M., Song, S., \& Pelli, D. G. (2007). Amblyopic reading is crowded Journal of Vision, 7(2), 117.

Manning, C., Jones, P. R., Dekker, T. M., \& Pellicano, E. (2018). Psychophysics with children: Investigating the effects of attentional lapses on threshold estimates. Attention, Perception, \& Psychophysics, 80, 1311-1324.

Mareschal, I., Morgan, M. J., \& Solomon, J. A. (2010). Cortical distance determines whether flankers cause crowding or the tilt illusion. Journal of Vision, 10(8):13, 1-14.

Martelli, M., Di Filippo, G., Spinelli, D., \& Zoccolotti, P. (2009). Crowding, reading, and developmental dyslexia. Journal of Vision, 9(4), 1-18.

McKee, S. P., Levi, D. M., \& Movshon, J. A. (2003). The pattern of visual deficits in amblyopia. Journal of Vision, 3 (5), 5-5.

Miles, W. (1928). Ocular dominance: Methods and results. Psychological Bulletin, 25, 155-156.

Motter, B. C. (2009). Central V4 Receptive Fields Are Scaled by the V1 Cortical Magnification and Correspond to a Constant-Sized Sampling of the V1 Surface. Journal of Neuroscience, 29(18), 5749-5757.

Motter, B. C., \& Simoni, D. A. (2007). The roles of cortical image separation and size in active visual search performance. Journal of Vision, 7(2(6)), 1-15.

Moutsiana, C., de Haas, B., Papageorgiou, A., van Dijk, J. A., Balraj, A., Greenwood, J. A., et al. (2016). Cortical idiosyncrasies predict the perception of object size. Nature Communications, 7(12110), 1-12.

Parkes, L., Lund, J., Angelluci, A., Solomon, J. A., \& Morgan, M. (2001). Compulsory averaging of crowded orientation signals in human vision. Nature Neuroscience, 4(7), 6.

Pascal, E., \& Abadi, R. V. (1995). Contour interaction in the presence of congenital nystagmus. Vision Research, 35(12), 1785-1789.

Pelli, D. G. (1997). The VideoToolBox software for visual psychophysics: Transforming numbers into movies. Spatial Vision, 10(4), 437-442.

Pelli, D. G., Palomares, M., \& Majaj, N. J. (2004). Crowding is unlike ordinary masking: Distinguishing feature integration from detection. Journal of Vision, 4(12), 12-12.

Petrov, Y., \& Meleshkevich, O. (2011). Asymmetries and idiosyncratic hot spots in crowding. Vision Research, 51(10), 1117-1123.

Pouget, A., Dayan, P., \& Zemel, R. (2000). Information processing with population codes. Nature Reviews Neuroscience, 1(125-132).

Pugh, M. (1958). Visual distortions in amblyopia. British Journal of Ophthalmology, 42, 449-460.

Rosenholtz, R., Yu, D., \& Keshvari, S. (2019). Challenges to pooling models of crowding: Implications for visual mechanisms. Journal of Vision, 19(7), 15.

Schiller, P. H., Finlay, B. L., \& Volman, S. F. (1976). Quantitative studies of single-cell properties in monkey striate cortex $\|$. Orientation specificity and ocular dominance. Journal of Neurophysiology, 38(6), 1320-1333.

Sereno, M. I., Dale, A. M., Reppas, J. B., Kwong, K. K., Belliveau, J. W., Brady, T. J., et al. (1995). Borders of multiple visual areas in humans revealed by functional magnetic resonance imaging. Science, 268, 889-893. 
Shooner, C., Hallum, L. E., Kumbhani, R. D., Ziemba, C. M., Garcia-Marin, V., Kelly, J. G., et al. (2015). Population representation of visual information in areas V1 and V2 of amblyopic macaques. Vision Research, 114, 56-67.

Simons, K. (1983). Visual acuity norms in young children Survey of Ophthalmology, 28(2), 84-92.

Sireteanu, R., Lagreze, W.-D., \& Constantinescu, D. H. (1993). Distortions in two-dimensional visual space perception in strabismic observers. Vision Research, 33(5-6), 677-690.

Skoczenski, A. M., \& Norcia, A. M. (2002). Late maturation of visual hyperacuity. Psychological Science, 13(6), 537-541.

Sloan, L. L. (1959). New test charts for the measurement of visual acuity at far and near distances. American Journal of Ophthalmology, 48(6), 807-813.

Song, H., Levi, D. M., \& Pelli, D. G. (2014). A double dissociation of the acuity and crowding limits to letter identification, and the promise of improved visual screening. Journal of Vision, 14(5), 1-37.

Strasburger, H. (2005). Unfocused spatial attention underlies the crowding effect in indirect form vision. Journal of Vision, 5(11), 1024-1037.

Strasburger, H., Harvey, L. O. J., \& Rentschler, I. (1991). Contrast thresholds for identification of numeric characters. Perception \& Psychophysics, 49(6), 495-508.

Strasburger, H., Rentschler, I., \& Jüttner, M. (2011). Peripheral vision and pattern recognition: A review. Journal of Vision, 11(5), 1-82.

Tailor, V. K., Theodorou, M., Dahlmann-Noor, A. H., Dekker, T. M., \& Greenwood, J. A. (2021). Eye movements elevate crowding in congenital idiopathic nystagmus. bioRxiv, 2021.2001 .2016 .426927$.

Toet, A., \& Levi, D. M. (1992). The two-dimensional shape of spatial interaction zones in the parafovea. Vision Research, 32(7), 1349-1357.

Treutwein, B. (1995). Adaptive psychophysical procedures Vision Research, 35(17), 2503-2522.

Tripathy, S. P., \& Cavanagh, P. (2002). The extent of crowding in peripheral vision does not scale with target size. Vision Research, 42, 2357-2369.

van den Berg, R., Roerdink, J. B. T. M., \& Cornelissen, F. W. (2010). A neurophysiologically plausible population code model for feature integration explains visual crowding. PLoS Computational Biology, 6(1), e1000646.

Van Essen, D. C., Newsome, W. T., \& Maunsell, J. H. (1984). The visual field representation in the striate cortex of the macaque monkey: Asymmetries, anisotropies, and individual variability. Vision Research, 24, 429-448.

Watson, A. B., \& Pelli, D. G. (1983). Quest: A Bayesian adaptive psychometric method. Perception \& Psychophysics, 33(2), 113-120.

Whitney, D., \& Levi, D. M. (2011). Visual crowding: A fundamental limit on conscious perception and object recognition. Trends in Cognitive Sciences, 15(4), 160-168.

Wilkinson, F., Wilson, H. R., \& Ellemberg, D. (1997). Lateral interactions in peripherally viewed texture arrays. Journal of the Optical Society of America A, 14(9), 2057-2068.

Witton, C., Talcott, J. B., \& Henning, B. (2017). Psychophysical measurements in children: Challenges, pitfalls, and considerations. PeerJ, 5, e3231.

Wolford, G. (1975). Perturbation model for letter identification. Psychological Review, 82, 184-199.

Yong, K. X. X., Shakespeare, T. J., Cash, D., Henley, S. M. D., Nicholas, J. M., Ridgway, G. R., et al. (2014). Prominent effects and neural correlates of visual crowding in a neurodegenerative disease population. Brain, 137(12), 3284-3299.

Zhang, B., Zheng, J., Watanabe, I., Maruko, I., Bi, H., Smith, E. L., et al. (2005). Delayed maturation of receptive field center/surround mechanisms in V2. Procedings of the National Academy of Sciences of the United States of America, 102(16), 5862-5867. 


\section{Appendices}

Appendix A: Clinical details of children with typical vision and amblyopia

\begin{tabular}{|c|c|c|c|c|c|}
\hline Observer & $\begin{array}{l}\text { Age } \\
\text { (months) }\end{array}$ & Gender & Refractive error & $\begin{array}{l}\text { logMAR } \\
\text { acuity }\end{array}$ & $\begin{array}{l}\text { TNO } \\
\text { stereo }\end{array}$ \\
\hline $\mathrm{T} 1$ & 69 & $\mathrm{M}$ & $\begin{array}{l}\text { L: Plano } \\
\text { R: Plano }\end{array}$ & $\begin{array}{l}\text { L: } 0.00 \\
\text { R: } 0.00\end{array}$ & $60 "$ \\
\hline T2 & 78 & $\mathrm{~F}$ & $\begin{array}{l}\text { L: Plano } \\
\text { R: Plano }\end{array}$ & $\begin{array}{l}\text { L: } 0.00 \\
\text { R: } 0.00\end{array}$ & $60 "$ \\
\hline T3 & 82 & $\mathrm{~F}$ & $\begin{array}{l}\text { L: Plano } \\
\text { R: Plano }\end{array}$ & $\begin{array}{l}\text { L: } 0.00 \\
\text { R: } 0.00\end{array}$ & $60 "$ \\
\hline T4 & 82 & $\mathrm{~F}$ & $\begin{array}{l}\text { L: Plano } \\
\text { R: Plano }\end{array}$ & $\begin{array}{l}\text { L: } 0.06 \\
\text { R: } 0.00\end{array}$ & $60 "$ \\
\hline T5 & 94 & $\mathrm{M}$ & $\begin{array}{l}\text { L: Plano } \\
\text { R: Plano }\end{array}$ & $\begin{array}{l}\text { L: } 0.00 \\
\text { R: } 0.00\end{array}$ & $60 "$ \\
\hline T6 & 88 & $M$ & $\begin{array}{l}\text { L: Plano } \\
\text { R: Plano }\end{array}$ & $\begin{array}{l}\text { L: } 0.00 \\
\text { R: } 0.00\end{array}$ & $60 "$ \\
\hline T7 & 73 & $M$ & $\begin{array}{l}\text { L: Plano } \\
\text { R: Plano }\end{array}$ & $\begin{array}{l}\text { L: } 0.10 \\
R: 0.04\end{array}$ & $120 "$ \\
\hline T8 & 60 & M & $\begin{array}{l}\text { L: Plano } \\
\text { R: Plano }\end{array}$ & $\begin{array}{l}\text { L: } 0.10 \\
\text { R: } 0.04\end{array}$ & $60 "$ \\
\hline T9 & 54 & $M$ & $\begin{array}{l}\mathrm{L}:+0.50 \mathrm{DS} \\
\mathrm{R}:+1.00 \mathrm{DS}\end{array}$ & $\begin{array}{l}\text { L: } 0.16 \\
\text { R: } 0.18\end{array}$ & $120 "$ \\
\hline T10 & 73 & $M$ & $\begin{array}{l}\text { L: }+0.50 \mathrm{DS} \\
\mathrm{R}:+1.00 \mathrm{DS}\end{array}$ & $\begin{array}{l}\text { L: } 0.10 \\
\text { R: } 0.20\end{array}$ & $60 "$ \\
\hline T11 & 84 & M & $\begin{array}{l}\mathrm{L}:+0.25 \mathrm{DS} \\
\mathrm{R}:+0.25 \mathrm{DS}\end{array}$ & $\begin{array}{l}\text { L: } 0.00 \\
\text { R: } 0.00\end{array}$ & $60 "$ \\
\hline T12 & 36 & $\mathrm{~F}$ & $\begin{array}{l}\text { L: Plano } \\
\text { R: Plano }\end{array}$ & $\begin{array}{l}\text { L: } 0.00 \\
\text { R: } 0.10\end{array}$ & $60 "$ \\
\hline T13 & 93 & $\mathrm{M}$ & $\begin{array}{l}\mathrm{L}:-0.75 /-1.00 \times 50^{\circ} \\
\mathrm{R}:-3.00 \mathrm{DS}\end{array}$ & $\begin{array}{l}\text { L: } 0.12 \\
\text { R: } 0.20\end{array}$ & $120 "$ \\
\hline T14 & 70 & $M$ & $\begin{array}{l}\text { L: Plano } \\
\text { R: Plano }\end{array}$ & $\begin{array}{l}\text { L: }-0.10 \\
R:-0.10\end{array}$ & $60 "$ \\
\hline T15 & 73 & M & $\begin{array}{l}\text { L: Plano } \\
\text { R: Plano }\end{array}$ & $\begin{array}{l}\text { L: } 0.00 \\
\text { R: } 0.00\end{array}$ & $60 "$ \\
\hline T16 & 73 & $\mathrm{M}$ & $\begin{array}{l}\text { L: Plano } \\
\text { R: Plano }\end{array}$ & $\begin{array}{l}\text { L: } 0.00 \\
\text { R: } 0.00\end{array}$ & $60 "$ \\
\hline T17 & 89 & $M$ & $\begin{array}{l}\text { L: Plano } \\
\text { R: Plano }\end{array}$ & $\begin{array}{l}\text { L: } 0.00 \\
\text { R: } 0.00\end{array}$ & $60 "$ \\
\hline T18 & 61 & $\mathrm{~F}$ & $\begin{array}{l}\text { L: Plano } \\
\text { R: Plano }\end{array}$ & $\begin{array}{l}\mathrm{L}:-0.10 \\
\mathrm{R}:-0.10\end{array}$ & $60 "$ \\
\hline T19 & 74 & $\mathrm{~F}$ & $\begin{array}{l}\text { L: Plano } \\
\text { R: Plano }\end{array}$ & $\begin{array}{l}\text { L: } 0.02 \\
\text { R: } 0.10\end{array}$ & $60 "$ \\
\hline T20 & 56 & $F$ & $\begin{array}{l}\text { L: Plano } \\
\text { R: Plano }\end{array}$ & $\begin{array}{l}\text { L: } 0.00 \\
\text { R: } 0.00\end{array}$ & $60 "$ \\
\hline
\end{tabular}

Table A1. Clinical details of children included in the group with typical vision $(\mathrm{N}=20)$.

Age is reported in months. Optical correction includes cylindrical and spherical values (DS = dioptres) with the appropriate axes for each eye ( $L=$ left eye, $R=$ right eye). logMAR acuity is also reported for each eye. Results from the TNO stereo-acuity test and are reported in seconds of arc. 


\begin{tabular}{|c|c|c|c|c|c|c|}
\hline Observer & $\begin{array}{l}\text { Age } \\
\text { (months) }\end{array}$ & Gender & $\begin{array}{l}\text { Ocular } \\
\text { alignment }\end{array}$ & Refractive error & $\begin{array}{l}\text { logMAR } \\
\text { acuity }\end{array}$ & $\begin{array}{l}\text { TNO } \\
\text { stereo. }\end{array}$ \\
\hline A1 & 89 & $M$ & L ET $20^{\Delta}$ & $\begin{array}{l}\mathrm{L}:+5.25 \mathrm{DS} \\
\mathrm{R}:+1.75 \mathrm{DS}\end{array}$ & $\begin{array}{l}\mathrm{L}: 0.32 \\
\mathrm{R}:-0.06\end{array}$ & Nil \\
\hline $\mathrm{A} 2$ & 63 & $M$ & L ET $30^{\Delta}$ & $\begin{array}{l}\mathrm{L}:+6.5 /-1.25 \times 175^{\circ} \\
\mathrm{R}:+4.5 /-0.5 \times 5^{\circ}\end{array}$ & $\begin{array}{l}\text { L: } 0.36 \\
\text { R: } 0.1\end{array}$ & Nil \\
\hline A3 & 100 & $\mathrm{~F}$ & $\mathrm{RET} 40^{\Delta}$ & $\begin{array}{l}\mathrm{L}:+2.25 /-0.25 \times 180^{\circ} \\
\mathrm{R}:+1.25 /-2.00 \times 15^{\circ}\end{array}$ & $\begin{array}{l}\text { L: } 0.04 \\
\text { R: } 0.32\end{array}$ & Nil \\
\hline A4 & 62 & $\mathrm{M}$ & $\mathrm{R} E T 35^{\Delta}$ & $\begin{array}{l}\mathrm{L}:+2.00 \mathrm{DS} \\
\mathrm{R}:+2.50 /-5.00 \times 180^{\circ}\end{array}$ & $\begin{array}{l}\text { L: } 0.02 \\
\text { R: } 0.22\end{array}$ & Nil \\
\hline A5 & 67 & $\mathrm{~F}$ & L ET $6^{\Delta}$ & $\begin{array}{l}\mathrm{L}:+6.25 /-1.00 \times 180^{\circ} \\
R:+4.25 /-0.25 \times 180^{\circ}\end{array}$ & $\begin{array}{l}\text { L: } 0.46 \\
\text { R: } 0.00\end{array}$ & Nil \\
\hline A6 & 55 & $\mathrm{M}$ & $\begin{array}{l}\text { LET } 18^{\Delta} \\
\text { L/R } 9^{\Delta}\end{array}$ & $\begin{array}{l}\mathrm{L}:+2.00 /-1.00 \times 180^{\circ} \\
\mathrm{R}:+0.50 /-0.50 \times 180^{\circ}\end{array}$ & $\begin{array}{l}\mathrm{L}: 0.36 \\
\mathrm{R}: 0.00\end{array}$ & Nil \\
\hline A7 & 70 & $\mathrm{~F}$ & L ET $4^{\Delta}$ & $\begin{array}{l}\mathrm{L}:+7.00 /-0.50 \times 180^{\circ} \\
\mathrm{R}:+6.75 \mathrm{DS}\end{array}$ & $\begin{array}{l}\text { L: } 0.76 \\
\text { R: } 0.00\end{array}$ & Nil \\
\hline A8 & 37 & $\mathrm{~F}$ & $\mathrm{R} E T 30^{\Delta}$ & $\begin{array}{l}\mathrm{L}:+7.25 /-1.50 \times 5^{\circ} \\
\mathrm{R}:+8.00 /-1.50 \times 70^{\circ}\end{array}$ & $\begin{array}{l}\text { L: } 0.12 \\
\mathrm{R}: 1.10\end{array}$ & Nil \\
\hline A9 & 89 & $\mathrm{~F}$ & $\begin{array}{l}\text { n: RET } 25^{\Delta} \\
\text { d: R ET } 18^{\Delta}\end{array}$ & $\begin{array}{l}\mathrm{L}:+7.25 /-2.00 \times 170^{\circ} \\
\mathrm{R}:+7.75 /-1.00 \times 20^{\circ}\end{array}$ & $\begin{array}{l}\text { L: } 0.00 \\
\text { R: } 0.24\end{array}$ & Nil \\
\hline A10 & 76 & $\mathrm{~F}$ & $\begin{array}{l}\text { n: R ET } 16^{\Delta} \\
\text { d: R ET } 10^{\Delta}\end{array}$ & $\begin{array}{l}\mathrm{L}:+4.00 /+0.5 \times 180^{\circ} \\
\mathrm{R}:+4.50 /-0.5 \times 175^{\circ}\end{array}$ & $\begin{array}{l}\text { L: } 0.00 \\
\text { R: } 0.38\end{array}$ & Nil \\
\hline A11 & 77 & $\mathrm{M}$ & LET 35 & $\begin{array}{l}\mathrm{L}:+5.50 /-0.50 \times 180^{\circ} \\
\mathrm{R}:+3.00 /-0.50 \times 180^{\circ}\end{array}$ & $\begin{array}{l}\text { L: } 0.50 \\
\text { R: } 0.00\end{array}$ & Nil \\
\hline A12 & 86 & $\mathrm{~F}$ & $\begin{array}{l}\text { n: RET } 10^{\Delta} \\
\text { d: RET } 4^{\Delta}\end{array}$ & $\begin{array}{l}\mathrm{L}:+3.00 /-0.50 \times 180^{\circ} \\
\mathrm{R}:+5.00 /-1.00 \times 180^{\circ}\end{array}$ & $\begin{array}{l}\text { L: } 0.10 \\
\text { R: } 0.36\end{array}$ & Nil \\
\hline A13 & 64 & $\mathrm{~F}$ & $\mathrm{~L} X \mathrm{~T} 20^{\Delta}$ & $\begin{array}{l}\text { L: }+6.25 /-1.25 \times 180^{\circ} \\
\text { R: Plano }\end{array}$ & $\begin{array}{l}\text { L: } 0.50 \\
\text { R: } 0.00\end{array}$ & Nil \\
\hline A14 & 79 & M & $\mathrm{R}$ ET $8^{\Delta}$ & $\begin{array}{l}\text { L: } 0.50 /-0.25 \times 180^{\circ} \\
\text { R: } 5.50 /-0.75 \times 180^{\circ}\end{array}$ & $\begin{array}{l}\text { L: } 0.00 \\
\text { R: } 0.54\end{array}$ & $480 "$ \\
\hline A15 & 83 & $\mathrm{M}$ & L ET $45^{\Delta}$ & $\begin{array}{l}\mathrm{L}:+6.50 /-1.00 \times 180^{\circ} \\
\mathrm{R}:+4.75 /-1.25 \times 175^{\circ}\end{array}$ & $\begin{array}{l}\text { L: } 0.92 \\
\text { R: } 0.00\end{array}$ & Nil \\
\hline A16 & 70 & $\mathrm{~F}$ & $\mathrm{~L} X \mathrm{~T} 10^{\Delta}$ & $\begin{array}{l}\mathrm{L}:+6.00 \mathrm{DS} \\
\mathrm{R}:+6.00 \mathrm{DS}\end{array}$ & $\begin{array}{l}\text { L: } 0.22 \\
\text { R: } 0.00\end{array}$ & Nil \\
\hline A17 & 85 & $\mathrm{M}$ & L XT $25^{\Delta}$ & $\begin{array}{l}\mathrm{L}:+7.00 /-1.00 \times 180^{\circ} \\
\mathrm{R}:+1.25 /-0.25 \times 180^{\circ}\end{array}$ & $\begin{array}{l}\text { L: } 0.86 \\
\text { R: } 0.00\end{array}$ & Nil \\
\hline A18 & 73 & $M$ & L ET $40^{\Delta}$ & $\begin{array}{l}\mathrm{L}:+8.00 /-1.50 \times 70^{\circ} \\
\mathrm{R}:+7.50 /-1.00 \times 20^{\circ}\end{array}$ & $\begin{array}{l}\text { L: } 0.72 \\
\text { R: } 0.18\end{array}$ & Nil \\
\hline A19 & 45 & $\mathrm{~F}$ & LET $10^{\Delta}$ & $\begin{array}{l}\mathrm{L}:+7.50 /-1.00 \times 70^{\circ} \\
\mathrm{R}:+6.50 /-0.50 \times 180^{\circ}\end{array}$ & $\begin{array}{l}\mathrm{L}: 0.36 \\
\mathrm{R}: 0.12\end{array}$ & Nil \\
\hline A20 & 44 & $\mathrm{M}$ & L ET $20^{\Delta}$ & $\begin{array}{l}\mathrm{L}:+7.00 /-0.5 \times 20^{\circ} \\
\mathrm{R}:+6.00 /-1.00 \times 120^{\circ}\end{array}$ & $\begin{array}{l}\text { L: } 0.44 \\
R: 0.02\end{array}$ & Nil \\
\hline
\end{tabular}

Table A2. Clinical details of children with strabismic amblyopia included in the amblyopic group (N=20).

The "ocular alignment" column indicates the outcome of near (n) and distance (d) prism tests. ET = esotropia, XT = exotropia, $L / R=$ hypotropia. The degree of deviation is shown in prism dioptres $\left(^{\Delta}\right)$, with the amblyopic eye denoted as $L$ or R, and prism cover test results shown for both near (n) and distance (d) when required. Remaining columns are in the same format as Table A1 above. 


\section{Appendix B: Noise model simulations of group response-error distributions}

As described in the main text, a noise model was fit to the data in order to examine the response errors that would arise if crowding does not have a systematic effect on target appearance, but rather distorts or adds noise to the target orientation. Figure A1 shows the result of 1000 iterations of the best-fitting noise model, fit to group data. For adults, we depict here only the extreme eccentricities, with data for $2.5^{\circ}$ in panel $A$ and for $15^{\circ}$ in panel B. For unflanked targets, the model again captures the distribution of response errors well, given that the first stage of the model is identical to that of the pooling model. However, $30^{\circ}$ target-flanker differences, the responses clearly shift away from the target towards the flanker, whereas the simulated responses of the noise model remain centred on $0^{\circ}$. Similarly, although the noise model can simulate the peak of responses around the target with $90^{\circ}$ target-flanker differences, it fails to account for the secondary peak around the flanker orientation. This is even more apparent at $15^{\circ}$ eccentricity where the peak around the flanker orientation is higher than that around the target. Intermediate eccentricities show similar effects. The model similarly fails to account for these systematic errors in both children with typical vision (panel C) and those with amblyopia (panel D). An increase in the degree of random responses alone is clearly insufficient to account for these response-error distributions. 
A.

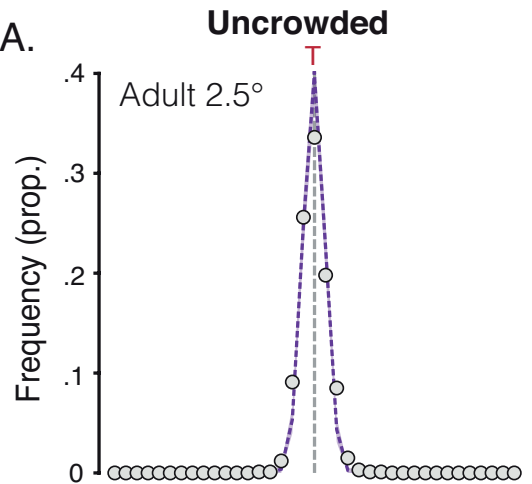

B.

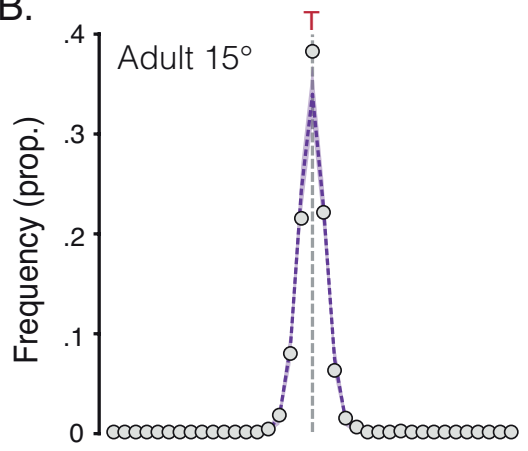

C.

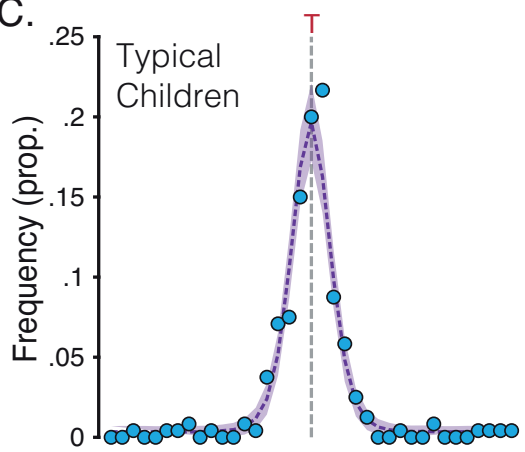

D.

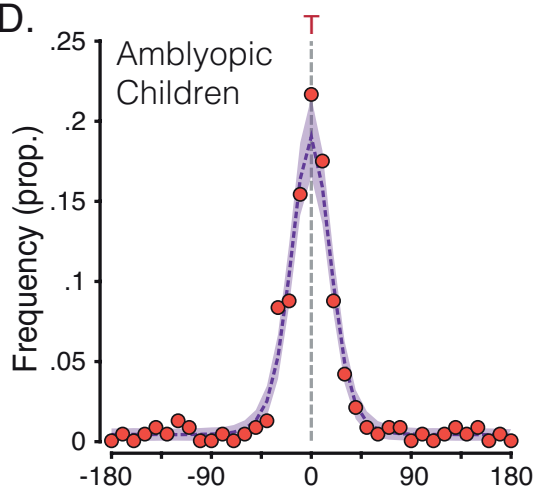

$30^{\circ}$ difference
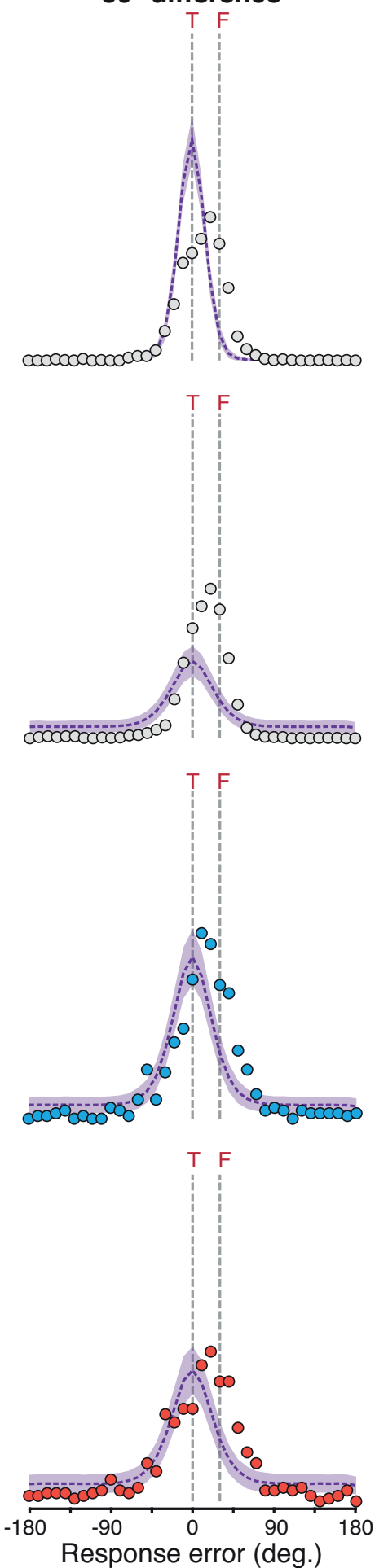
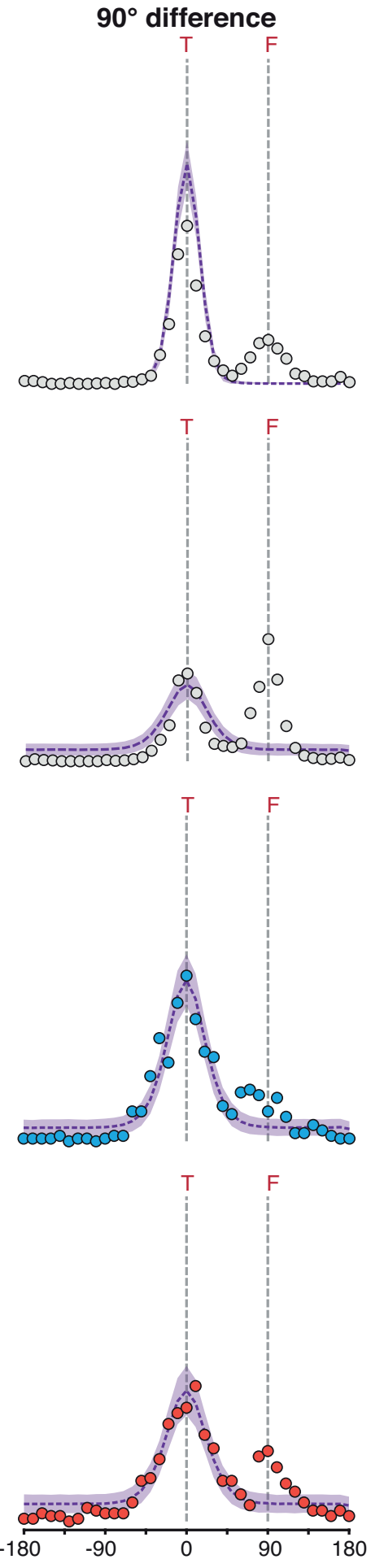

Figure A1. Distributions of mean response error and the simulated responses of the noise model.

A. Response error distributions at $2.5^{\circ}$ eccentricity, with mean values presented as light-grey dots. The purple dashed line plots the mean distribution of the noise model, with shaded areas plotting the $95 \%$ range of simulated distributions for 1000 model iterations. Dashed grey lines indicate the target location (' $T$ '), and for the conditions in which flankers were present, the flanker location ('F'). B. Response-error distributions at $15^{\circ}$ eccentricity and the best-fitting noise model, plotted as in panel A. C. Response-error distributions for children with typical vision, with mean values plotted in blue against the noise model. D. Response-error distributions for children with amblyopia, with mean values plotted in red against the noise model. 


\section{Appendix C: Best-fitting simulations of the models for individual data}

Both pooling and noise models were also fit to individual data, as outlined in the main text. Here we plot examples to illustrate variations in the success of these models. Figure A2 plots the responses of four adult observers. Panels $A$ and $B$ plot data from $2.5^{\circ}$ eccentricity. The first observer (S4) exhibited a large difference in the AIC for the two models. Although both perform equivalently for the uncrowded condition (given the identical first stage), their divergence is clear in the flanked conditions. In the $\pm 30^{\circ}$ condition, the pooling model successfully captures the shift of the response-error distribution towards the flanker, while the noise model remains centred on $0^{\circ}$. The secondary peak at $90^{\circ}$ is also missed by the noise model, as in the fits to group data. In contrast, observer S5 (panel B) exhibited a lower AIC value for the noise model at this eccentricity. Although the response error distribution in the $\pm 30^{\circ}$ condition is lower and broader than that of the unflanked condition, it fails to show a shift towards the flanker orientation of a magnitude equivalent to that seen in other observers and the group data. Consequently, although the slightly shifted distribution of the pooling model captures this pattern better than the noise model, this improvement is not significantly greater than the fit obtained by the noise model. This is similarly true in the $\pm 90^{\circ}$ condition where the secondary peak in response errors is absent. The models are thus difficult to distinguish for this observer given the lower rate of errors relative to that seen in other observers.

Similar patterns can be seen for the $15^{\circ}$ eccentricity data for two example observers in panels C and D. Observer S2 (panel C) exhibited a large difference in the AIC for the two models, driven by a clear pattern of systematic errors that is well captured by the pooling model. Contrast this with observer S1 (panel D) who showed one of the smallest AIC differences at this eccentricity. Systematic errors are less evident here, making the fit between the models harder to distinguish. Nonetheless, the shift at $30^{\circ}$ in particular was sufficient to produce a lower AIC value for the pooling model than the noise model, indicative of a better fit. 

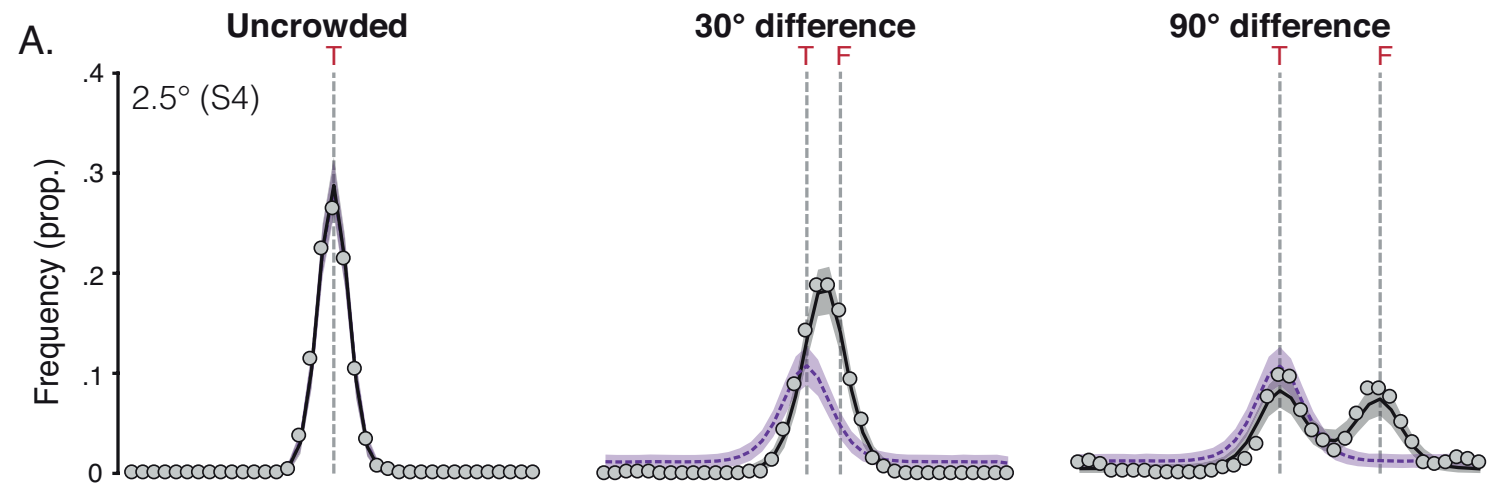

B.
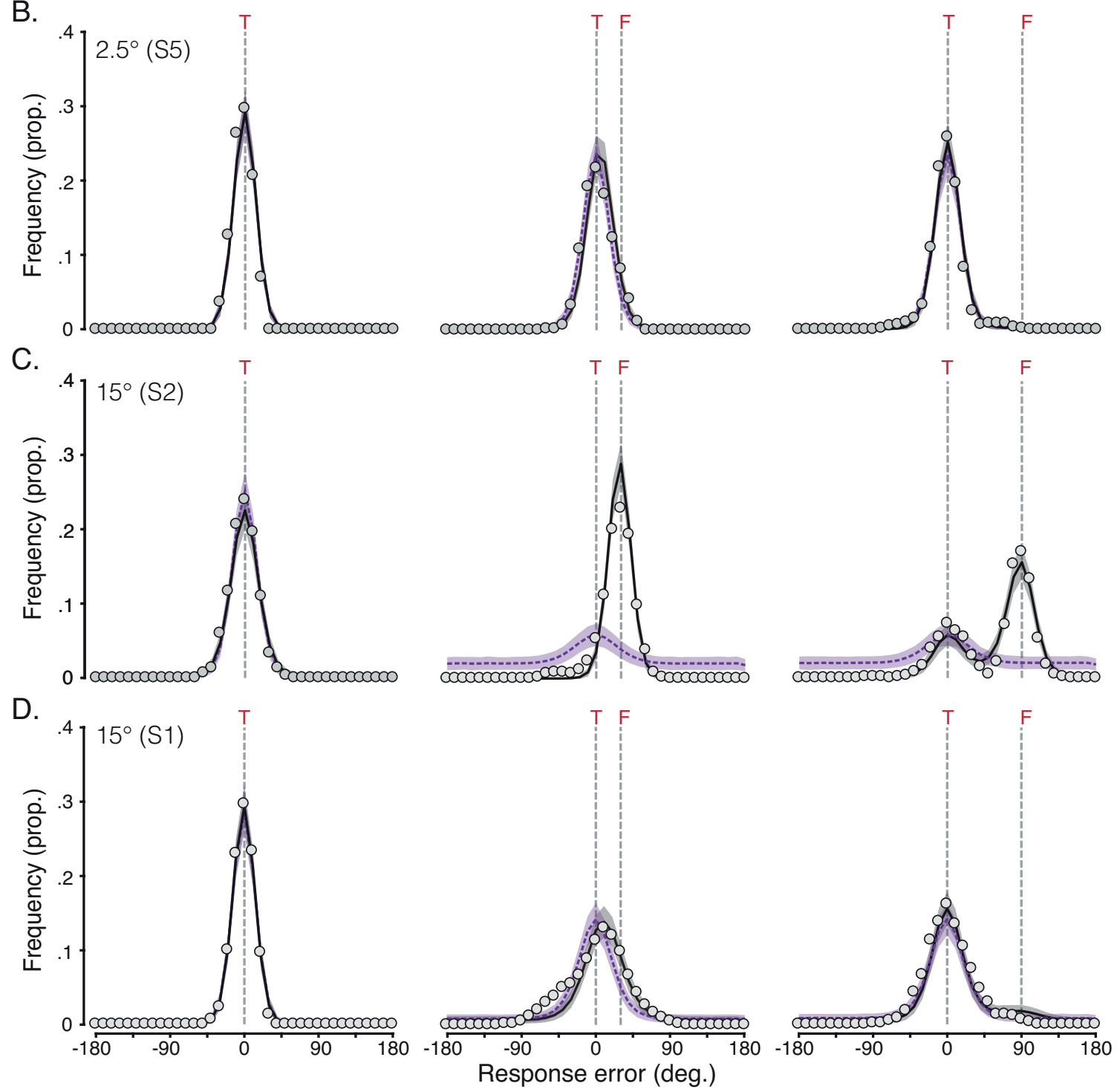

Figure A2. Individual data from the adult periphery and corresponding fits of the pooling and noise models.

A. Response error distributions for one individual (S4) at $2.5^{\circ}$ eccentricity, with mean values presented as light-grey dots. The black solid line plots the mean distribution of the pooling model, with the purple dashed line plotting the noise model. Shaded areas plot the 95\% range of simulated distributions. Dashed grey lines indicate the target location (' $T$ '), and for the conditions in which flankers were present, the flanker location ('F'). B. Response error distributions for another individual (S5) at $2.5^{\circ}$ eccentricity and associated model fits, plotted as in panel A. C-D. Response error distributions for individuals S2 (C) and S1 (D) at $15^{\circ}$ eccentricity and associated model fits, plotted as in panel A. 
Example fits to data from individual children are shown in Figure A3. Panels A and B plot example children with typical vision. The first (T6) had one of the largest AIC differences between the models, driven by the clear shift in their responses in the $\pm 30^{\circ}$ condition and the secondary peak in their responses in the $\pm 90^{\circ}$ condition. The noise model provides a poor account of these errors given that its distributions remain centred on $0^{\circ}$. Observer T2 however shows a lower degree of response errors, particularly in the $90^{\circ}$ condition, making the models more difficult to distinguish. Nonetheless, the small shift in the response-error distribution of the $30^{\circ}$ condition is sufficient to give a lower AIC value for the pooling model in this individual (and indeed in all control children).

Panels $C$ and $D$ show example individuals from the amblyopic group. Observer A11 (panel C) again shows a clear shift in response errors in the $\pm 30^{\circ}$ condition and a secondary peak in their responses in the $\pm 90^{\circ}$ condition, which the pooling model is well suited to describe. As above, the noise model provides a poor account of these errors given that its distributions remain centred on $0^{\circ}$. Observer A8 (panel D) differs in the sheer noisiness of their responses. Here there is clearly a distribution centred around the target, though there is little evidence for any change in these responses when flankers were added. As a result, the pooling and noise models provide equivalent fits to the data, and the penalisation of the pooling model for its greater number of parameters results in lower AIC values for the noise model. The failure of the pooling model in this case is thus driven by the noisy response-error distribution. This pattern was evident in 2 of the 20 observers in the amblyopic group, though the remainder had response error distributions that were more clearly systematic and thus better described by the pooling model. 

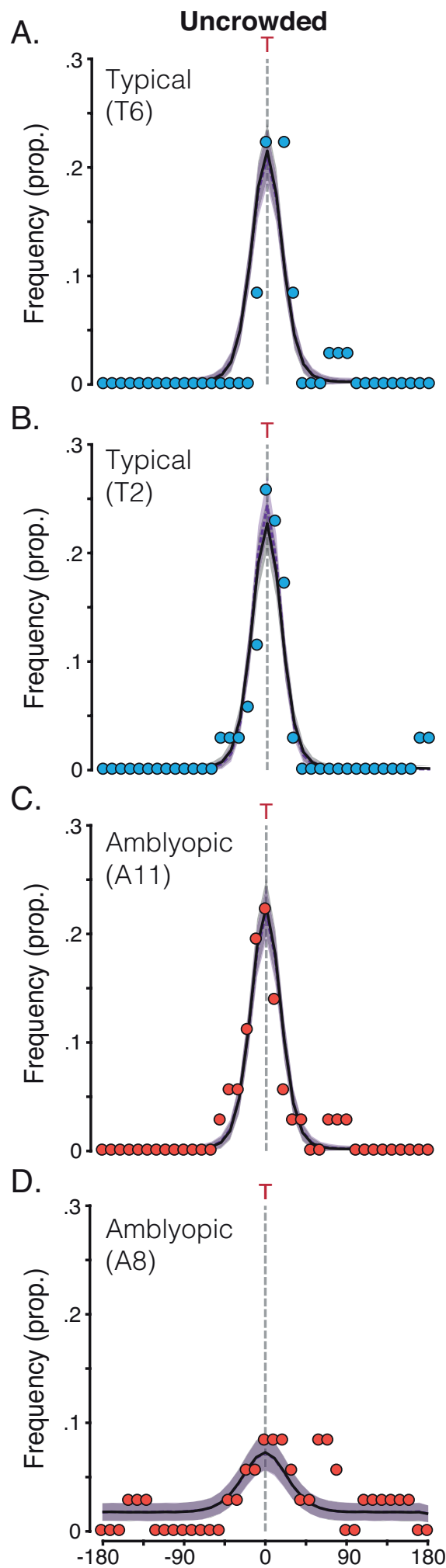
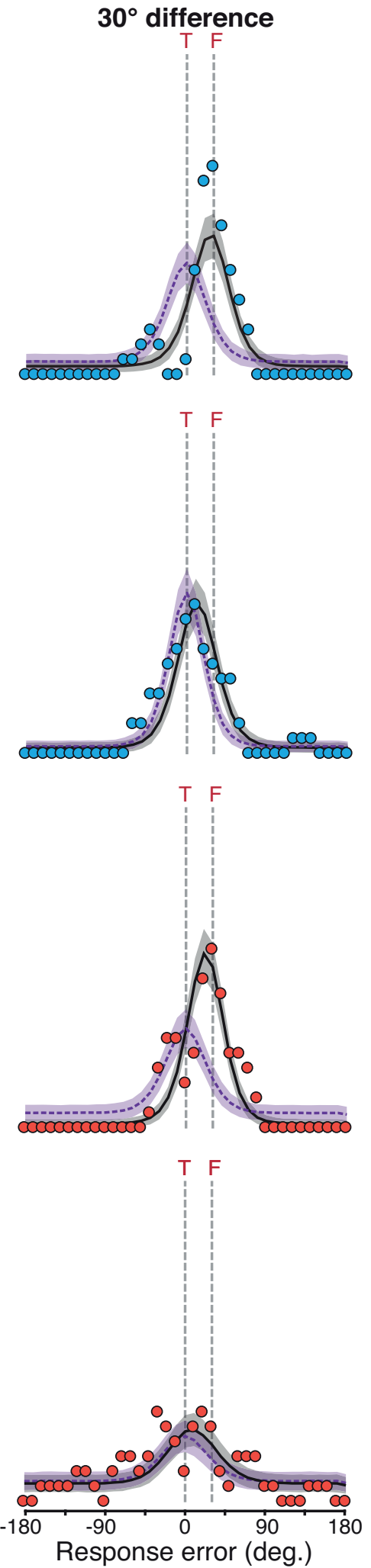
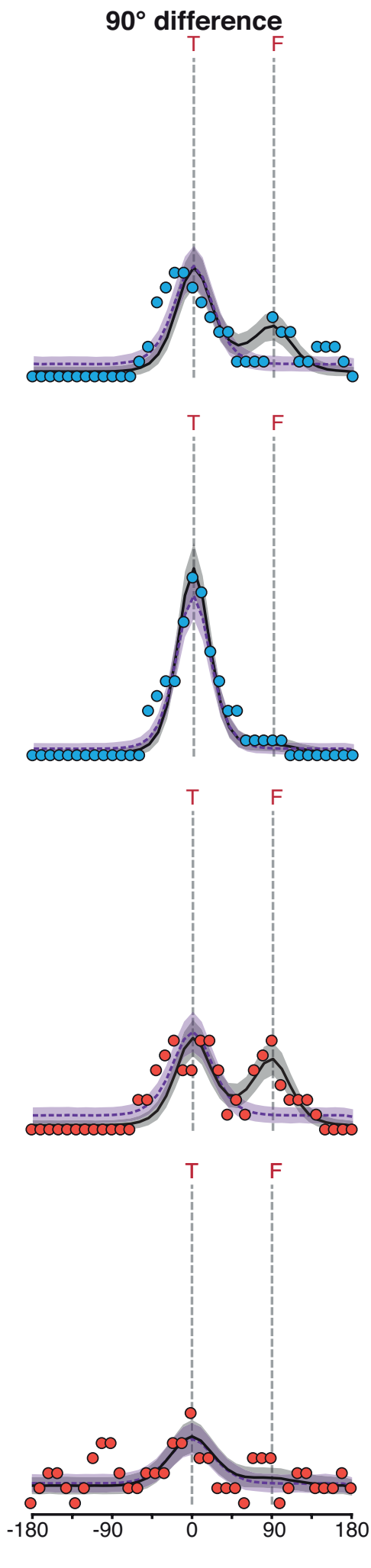

Figure A3. Individual data from children and corresponding fits of the pooling and noise models.

A. Response error distributions for one child with typical vision (T6), with mean values presented as blue dots. The black solid line plots the mean distribution of the pooling model, with the purple dashed line plotting the noise model. Shaded areas plot the $95 \%$ range of simulated distributions. Dashed grey lines indicate the target location ('T'), and for the conditions in which flankers were present, the flanker location ('F'). B. Response error distributions for another child with typical vision (T2) and associated model fits, plotted as in panel A. C-D. Response error distributions for two children with amblyopia - A11 (C) and A8 (D) and associated model fits, plotted as in panel A. 